\title{
Unidirectional and Bidirectional LSTM Models for Short-Term Traffic Prediction
}

\author{
Rusul L. Abduljabbar $\mathbb{D}^{1},{ }^{1}$ Hussein Dia, ${ }^{1}$ and Pei-Wei Tsai ${ }^{2}$ \\ ${ }^{1}$ Department of Civil and Construction Engineering, Swinburne University of Technology, Melbourne, Australia \\ ${ }^{2}$ Department of Computer Science and Software Engineering, Swinburne University of Technology, Melbourne, Australia
}

Correspondence should be addressed to Rusul L. Abduljabbar; rabduljabbar@swin.edu.au

Received 12 January 2021; Revised 31 January 2021; Accepted 20 February 2021; Published 26 March 2021

Academic Editor: Jinjun Tang

Copyright $\odot 2021$ Rusul L. Abduljabbar et al. This is an open access article distributed under the Creative Commons Attribution License, which permits unrestricted use, distribution, and reproduction in any medium, provided the original work is properly cited.

\begin{abstract}
This paper presents the development and evaluation of short-term traffic prediction models using unidirectional and bidirectional deep learning long short-term memory (LSTM) neural networks. The unidirectional LSTM (Uni-LSTM) model provides high performance through its ability to recognize longer sequences of traffic time series data. In this work, Uni-LSTM is extended to bidirectional LSTM (BiLSTM) networks which train the input data twice through forward and backward directions. The paper presents a comparative evaluation of the two models for short-term speed and traffic flow prediction using a common dataset of field observations collected from multiple freeways in Australia. The results showed BiLSTM performed better for variable prediction horizons for both speed and flow. Stacked and mixed Uni-LSTM and BiLSTM models were also investigated for 15minute prediction horizons resulting in improved accuracy when using 4-layer BiLSTM networks. The optimized 4-layer BiLSTM model was then calibrated and validated for multiple prediction horizons using data from three different freeways. The validation results showed a high degree of prediction accuracy exceeding $90 \%$ for speeds up to 60 -minute prediction horizons. For flow, the model achieved accuracies above $90 \%$ for 5 - and 10-minute prediction horizons and more than $80 \%$ accuracy for 15 - and 30 minute prediction horizons. These findings extend the set of AI models available for road operators and provide them with confidence in applying robust models that have been tested and evaluated on different freeways in Australia.
\end{abstract}

\section{Introduction}

Freeway short-term traffic prediction models have been researched extensively in the literature [1-8]. The strong interest in these models is that they can be used to provide road operators with predictive intelligence tools to help them optimize freeway operations and avoid traffic breakdowns. These models have been developed from a variety of theoretical backgrounds including statistical techniques and artificial intelligence (AI) methods based on neural networks [9]. With the development of big data and complex computational intelligence, AI methods can predict future traffic more accurately than statistical models. In particular, deep learning networks can represent traffic dynamic behaviour and have recently achieved massive success in time series modelling. An example of recent models is the unidirectional long short-term memory (Uni-LSTM) recurrent neural network and its extension bidirectional long short-term memory (BiLSTM). Previous research has shown that the Uni-LSTM model has an effective prediction in handling long-term dependencies as it remembers useful information from inputs that has already passed through using "additional gates" incorporated in its architecture [10-12]. However, in more recent years, a bidirectional LSTM (BiLSTM) model has been investigated which offers an additional training capability as the output layer receives information from past (backwards) and future (forward) instances simultaneously and it provides better prediction accuracy [13-16]. In this paper, we assess the performance of Uni-LSTM and BiLSTM for different time horizons using speed and flow field data for multiple freeways in Australia. The main research questions are as follows: 
(1) Will results be improved if speed and flow data are trained from both directions (forward direction and backward direction)?

(2) How adding layers or mixing both LSTM and BiLSTM as one model affects the model performance?

(3) If the model is trained and tested for one freeway, will it achieve a good accuracy if validated only (without retraining) on an independent dataset from a different freeway?

This paper aims to address these questions and demonstrate the feasibility of using advanced AI techniques based on deep learning Uni-LSTM and BiLSTM models to predict speed and flow for multiple prediction horizons. It provides a comparative performance analysis of both UniLSTM and BiLSTM based on a common dataset of field measurements. The models are developed using historical data extracted from sensors embedded in pavements on three freeways in Australia: the Pacific Motorway between Brisbane and the Gold Coast in Queensland, Tullamarine Freeway in Melbourne, and South Eastern Freeway in Melbourne. This paper also investigates whether additional layers of training improve prediction accuracies for both speed and flow. To our knowledge, there have been limited papers targeting the BiLSTM model for future traffic prediction, and this paper shows the robust performance of this extension of the Uni-LSTM model. Also, this paper validates the performance of a developed model on different freeways which makes this work a valuable contribution to knowledge in the intelligent transport systems and network operations fields. This provides road operators and transport agencies with confidence that they can apply these models on different freeways even if they have not embarked on comprehensive historical data collection efforts for the target freeways. This also helps them with reducing the cost of deployment of these algorithms by avoiding the need to preprocess new data and calibrate and validate new models which is a time-consuming undertaking that requires substantial resources and experienced and well-trained AI staff and specialists.

This paper is organized as follows: Section 2 provides a scan of previous research work. Section 3 presents the methodology including data collection and modelling frameworks. Section 4 presents the results of the comparative evaluation of different models. Section 5 presents the performance of stacked and mixed Uni-LSTM and BiLSTM models. Section 6 shows the model validation results, and finally, Section 7 presents the conclusions and future research directions.

\section{Literature Review}

The prediction and forecasting of short-term (1 to 60 minutes into the future) traffic conditions plays an important role in the success of intelligent transport systems (ITSs) such as travel information systems, adaptive traffic management systems, public transportation scheduling, and commercial vehicle operations [17-19]. Due to the wide body of literature on this topic, we focus the literature scan in this section on traffic prediction using LSTM models, which used field traffic data collected from inductive loop detectors, CCTV, probe vehicles, and incident reports. A comprehensive review of other models including those that used simulated data can be found in [17]. Increasingly, road operators have more confidence in models that have been developed using real-life data and hence our focus in this work on model development and evaluations using data from real-world environments.

Methodologies used for flow and speed prediction can be classified into two broad parametric and nonparametric approaches [20]. Examples of commonly used parametric methods include linear models such as autoregressive integrated moving average model (ARIMA) [21], seasonal ARIMA, i.e., SARIMA model [22], exponential smoothing model [23], and ARIMA with Kalman filter (KF) [24, 25]. These parametric methods perform poorly with dynamic traffic patterns, which limits their application in complex traffic prediction compared to nonparametric methods. Nonparametric methods are more capable of predicting a stochastic pattern of input traffic data and are better at handling noisy data.

With the recent advancements in machine learning, many models have shown a promising potential in solving nonlinear problems and handling long-term dependencies. Examples include LSTM and BiLSTM models. These models were previously used to forecast future traffic speeds [10], travel times [18], and traffic flows [11]. In one study, the long short-term memory (LSTM) structure was applied for future speed prediction and showed that it provides higher performance compared to classical methods [10]. Another study showed that using LSTM models is promising for irregular travel time prediction models as the error for 1-step-ahead prediction error is relatively small [18]. Other studies have shown that flow prediction using LSTM achieved high accuracy compared to other models for different prediction horizons [11]. Also, LSTM models have been developed in other studies on car-following models to predict acceleration and deceleration on different road hierarchies [26].

Short-term traffic flow using LSTM has also been investigated where the dependency relationships of time series data were fully considered, and experimental results showed a very good performance with an error of $5.4 \%$ when compared with other models [27]. In other studies, an endto-end deep learning model has been investigated to predict future traffic flows [28] where one BiLSTM layer was added, and the results showed that the model was capable of solving stochastic flow characteristics and overcoming overfitting problems [28]. Similarly, stacking BiLSTM and Uni-LSTM models were developed in another study to predict networkwide traffic speeds. The results showed that the stacked architecture outperforms both BiLSTM and Uni-LSTM models [29].

In another study, different models were developed that showed superior performance when using deeper BiLSTM layers for urban traffic prediction [30]. Other researchers have also used LSTM and RNN approaches for speed prediction models under various urban driving conditions with 
credible and accurate results [31]. LSTM and gated recurrent units (GRUs) were also applied in recent studies to predict the general condition of driving speed in consideration of the road geometry and temporal evolution of traffic demand. The results showed superior LSTM model performance compared to regression models [32]. Similarly, superior model performance has been shown from using LSTM and GRU models when compared to ARIMA and support vector regression (SVR) models for the track flow prediction [33].

In other studies, a variational long short-term memory encoder was examined to predict traffic flow which provided better prediction in comparison to other conventional methods used [34]. Similarly, a long short-term memorygenetic algorithm support vector regression (LSTMGASVR) short-term traffic flow prediction algorithm was reported to predict future traffic flows with better accuracies than LSTM, GRU, convolutional neural networks (CNNs), stacked autoencoder (SAE), ARIMA, and support vector regression (SVR) models tested in the same study [35].

Furthermore, LSTM models have also been developed for momentary traffic stream forecasts, which aim to help transport authorities in decision-making during rush hour for gridlock prediction since the model remembers information for longer periods of time than other models [36]. Also, the validity of LSTM models has been verified in studies on prediction of short-term traffic flow and were found to provide high prediction accuracies for flow data [37]. Other studies have documented superior performance when ARIMA and long short-term memory (LSTM) neural networks were combined for short-term traffic flow prediction [38]. In another recent study, type-2 fuzzy LSTM (T2F-LSTM) was developed for long-term traffic volume prediction and extraction of spatial-temporal characteristics of traffic volumes and was found to achieve high prediction accuracies [39].

In summary, a substantial number of studies in the literature have addressed short-term traffic prediction with robust LSTM models. However, only few studies have addressed the promising potential of BiLSTM models for traffic time series future prediction that consider the backward temporal dependencies. Another important contribution in this work is the comparison of stacked and mixed BiLSTM and LSTM layers for model accuracy improvement. In addition, this paper discusses the model applicability when being developed on parameters of one location and validated only (without retraining) on different locations. Furthermore, the models are developed using field data that comprised diverse and complex traffic characteristics (including peak, nonpeak, weekday, weekend, incident, and nonincident data). Another important factor in this work is that the data used for model development have been methodically screened, preprocessed, and validated in a large number of previous studies [40,41].

\section{Study Methodology}

This section of the paper presents the study methodology including data collection, model development, evaluation tests, and analyses.
3.1. Data for Model Development. Neural network applications require large amounts of data that are needed for model development $[41,42]$. The data are typically divided into a training dataset used for model calibration and a testing dataset used for model verification. The validity of the model is tested on an independent dataset not used in model training, referred to as the testing dataset. In this research, the data used for model development included traffic speed and flow measurements collected from sensors installed on a number of freeways in Australia. The data were collected over a number of years and time periods including peak, nonpeak, weekday, and weekend conditions. Another unique characteristic of the data is that they include incident traffic conditions which are usually difficult to capture for model development. Such incidents, which include road crashes, broken down vehicles, and similar nonrecurrent events, typically result in a significant capacity reduction of the freeway and last for long durations. Including these data in model training and validation improves the robustness of the prediction models.

3.1.1. Dataset 1: Pacific Motorway, Queensland. This dataset was collected from a section of the Pacific Motorway between Brisbane and the Gold Coast in Queensland [40]. The length of the section is around $1.5 \mathrm{~km}$. Speed and flow data were gathered from 4 detection stations (S0-S3) which include inductive loop sensors installed at approximately $500 \mathrm{~m}$ interval as shown in Figure 1. These data were collected for a period of 5 hours ( 2 hours peak and 3 hours offpeak traffic conditions). The data comprised normal traffic conditions that did not include any incidents. A total of 1,667 observations were gathered. For this study, the data were divided into 1000 observations for training ( $60 \%$ of the total dataset) and 667 observations for testing ( $40 \%$ of the total dataset).

3.1.2. Dataset 2: Tullamarine Freeway, Melbourne. This dataset was collected from a section of the Tullamarine Freeway in Melbourne as shown in Figure 2. The freeway connects the city to the airport and the northern suburbs and is considered one of the busiest roads in Melbourne. The data comprised lane-by-lane loop-detector data consisting of speed, flow, and occupancy measurements in 1-minute cycles [41]. Detector station spacing ranged between $450 \mathrm{~m}$ and $1070 \mathrm{~m}$, with an average spacing of about $580 \mathrm{~m}$ for the 14 detector stations. A total of 21,123 observations were gathered, and for this research, they were divided into $60 \%$ for training (12,674 observations) and $40 \%$ for testing $(8,449$ observations). This dataset was unique in that it comprised a total of 75 incidents that occurred on the freeway under variable traffic conditions. While such data make it very challenging to be able to predict future conditions (because of the randomness of incidents), they are most useful as they allow the prediction algorithms to be trained on a wide range of traffic data including both incident and nonincident conditions. 


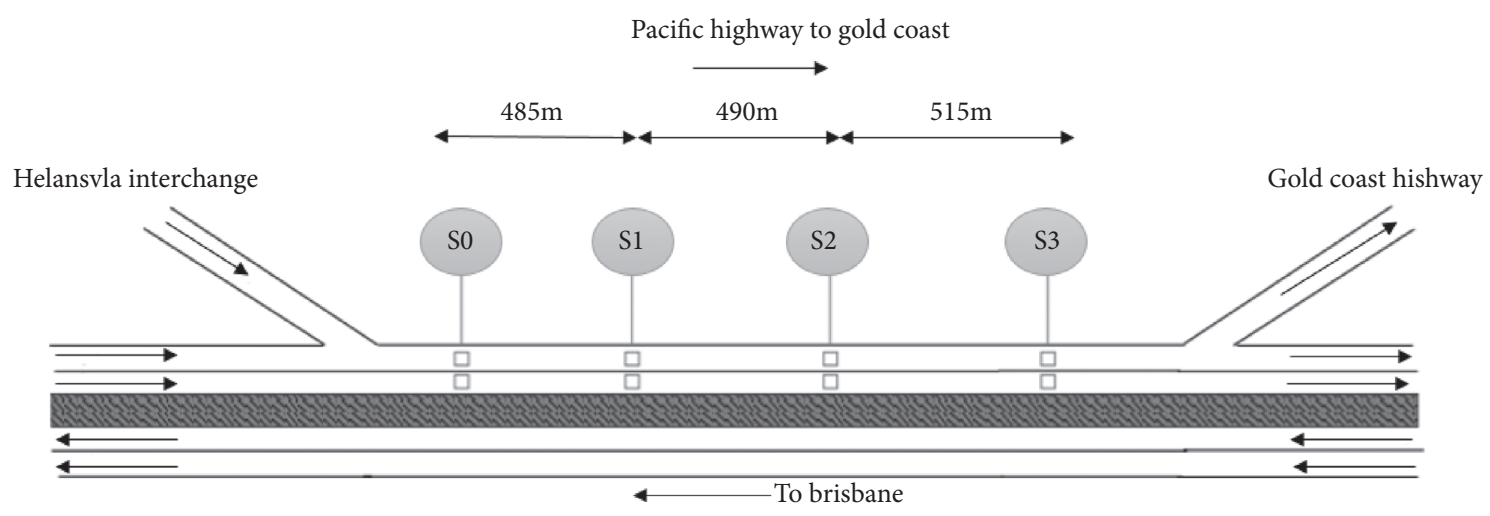

FIgure 1: Schematic of the Pacific Motorway section used for collecting dataset 1 [40].

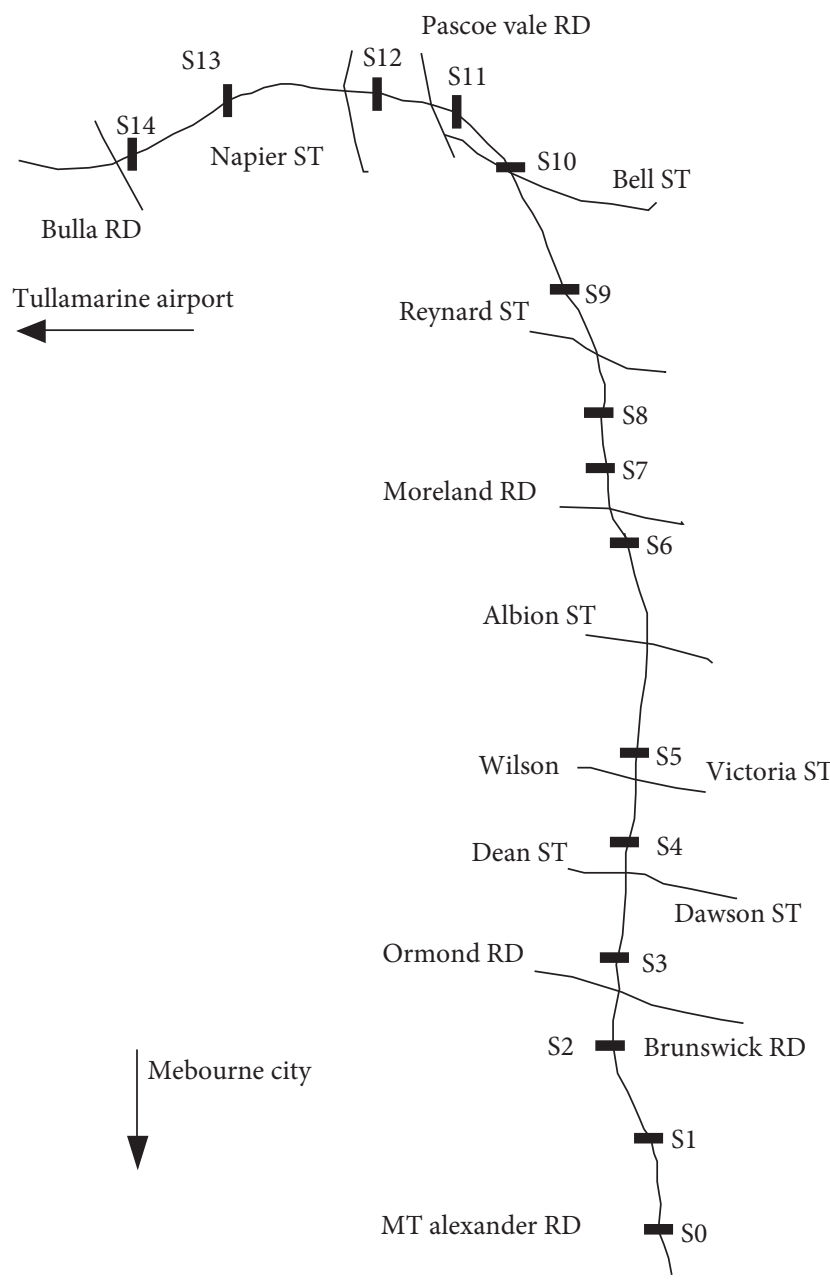

FIgURE 2: Schematic diagram of the Tullamarine Freeway used for collecting dataset 2 [41].

3.1.3. Dataset 3: South Eastern Freeway, Melbourne. This dataset was collected from a section of the South Eastern Freeway in Melbourne as shown in Figure 3. The data consisted of speed and flow measurements in 1-minute cycles, also collected from inductive loops embedded in the pavement. A total of 3,147 observations were gathered. For this work, the data were divided into $60 \%$ for training (1,888 observations) and $40 \%$ for testing (1,259 observations). Similarly, these data included a total of 25 incidents which would allow the prediction algorithm to be trained on a variety of traffic data including incident and nonincident conditions.

The data from the two Melbourne freeways are also important in that each freeway carries more than 100,000 vehicles per day. The incident data collected from these freeways (100 in total) had varying characteristics that included a representative range of incidents on freeways. For example, four incidents resulted in blocking one lane of traffic, 77 resulted in blocking 2 lanes, and 19 resulted in blocking three lanes. Five of the incidents occurred during low-flow conditions (below $700 \mathrm{vphpl}$ ), 58 during heavyflow conditions (above $1550 \mathrm{vphpl}$ ), and 37 during moderate flow conditions. Twenty-five incidents also occurred during peak-hour traffic conditions. As for the distribution of incident duration, 26 incidents lasted for less than 30 minutes; 32 lasted between 30 and 60 minutes; 30 lasted between 60 and 90 minutes; and 12 lasted more than 90 minutes [41].

Samples of speed and flow data for the three freeways are shown in Figures 4 and 5, respectively. These figures represent a small portion of the data for illustrative purposes. Figure 4 shows speed patterns in $\mathrm{km} / \mathrm{h}$ during AM peak between 5:30 and 8:00 AM. The figure demonstrates that the South Eastern Freeway is the most congested freeway with a speed lower than $20 \mathrm{~km} / \mathrm{h}$ followed by the Pacific Motorway and Tullamarine Freeway. As for Figure 5, the flow patterns are illustrated in veh/h for the period from $9 \mathrm{AM}$ to $12 \mathrm{PM}$. The figure shows that each freeway behaves differently during the same period of time as the flow ranges between 7 and 56 vehicles per hour for the three freeways. In summary, the reallife datasets used in this study are considered to be one of the most diverse and representative field traffic datasets available particularly in the Australian context. They are also unique in that they have been meticulously screened, cleaned, preprocessed, and validated in a large number of studies [15].

3.2. Modelling Framework. Unidirectional LSTM has received considerable attention in recent years for its superior performance compared to the state-of-the-art recurrent neural networks (RNNs). Even though RNNs provide good accuracy, they have been found to underperform for longterm memory as RNNs are unable to use information from distant past. Also, LSTM can learn patterns with long 


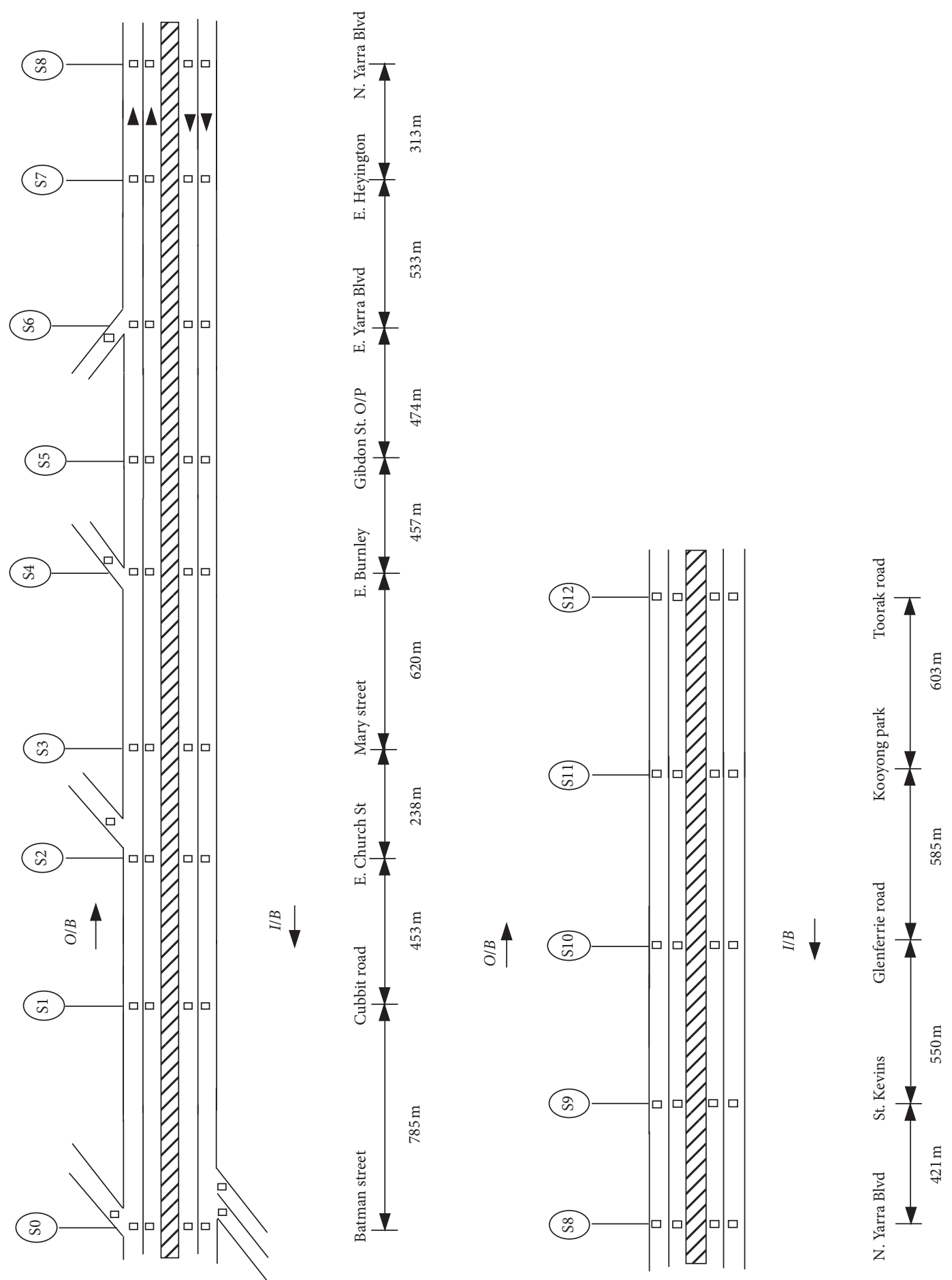

Figure 3: Schematic of the South Eastern Freeway.

dependencies when compared with traditional RNNs that are not able to function for long-term patterns. Therefore, LSTM has generally been found to outperform RNNs in time series data forecasting [43]. The inclusion of additional data training has resulted in some model extensions of LSTM, now known as bidirectional LSTM (BiLSTM). This model trains the input time series data twice through forward and backward directions. The architectures of these models are presented in Figures 6 and 7.
In these models, the following formulae are used to calculate the predicted values:

$$
\begin{aligned}
\text { input gate }\left(I_{t}\right) & =\sigma_{g}\left(W_{i} X_{t}+R_{i} h_{t-1}+b_{i}\right), \\
\text { forget gate }\left(f_{t}\right) & =\sigma_{g}\left(W_{f} X_{t}+R_{f} h_{t-1}+b_{f}\right), \\
\text { cell candidate }\left(C_{t}\right) & =\sigma_{c}\left(W_{c} X_{t}+R_{c} h_{t-1}+b_{c}\right), \\
\text { output gate }\left(o_{t}\right) & =\sigma_{g}\left(W_{o} X_{t}+R_{o} h_{t-1}+b_{o}\right),
\end{aligned}
$$




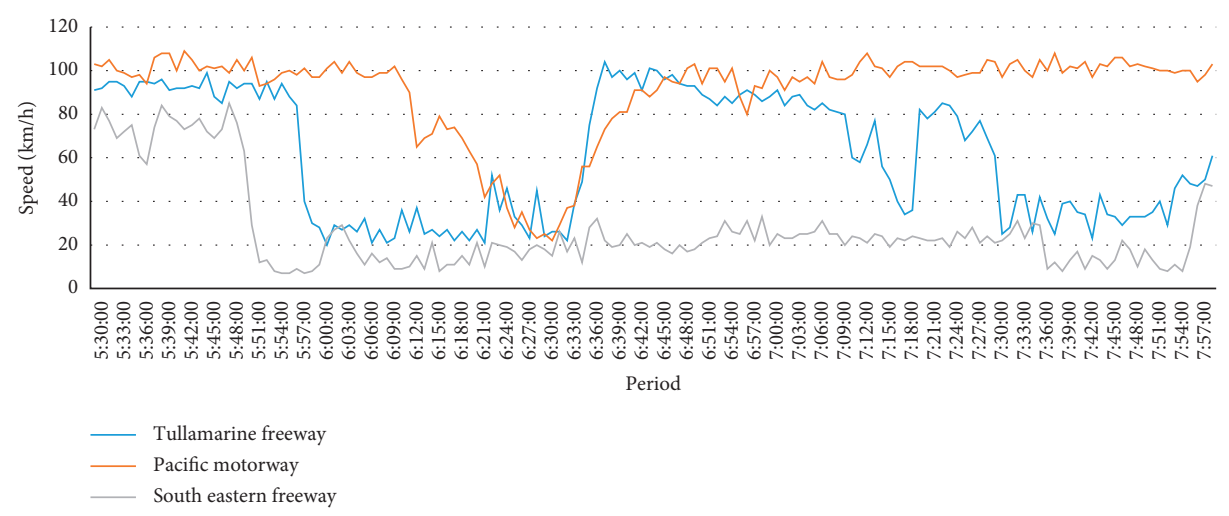

FIGURE 4: Samples of speed data for the three freeways.

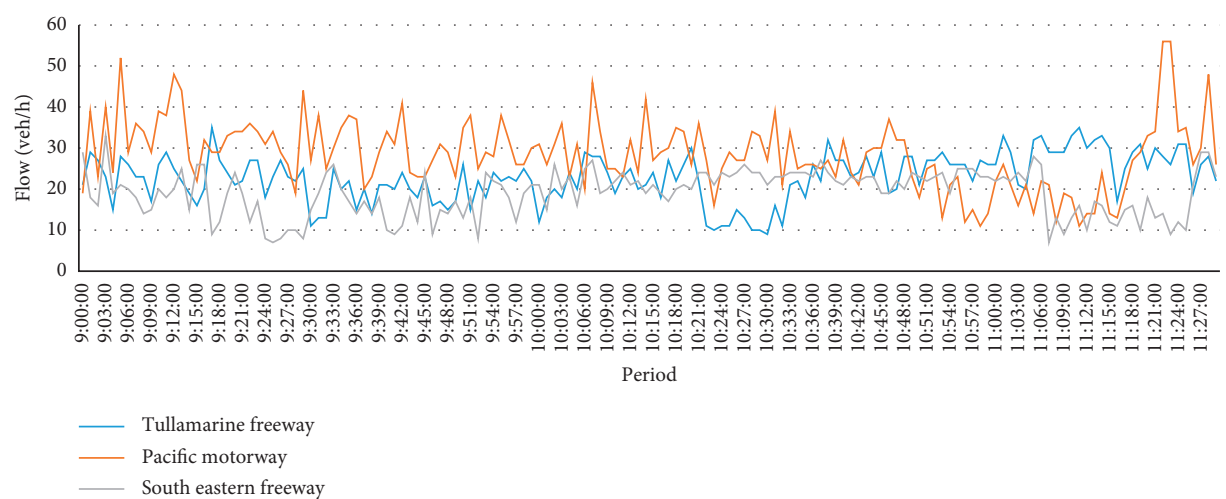

FIGURE 5: Samples of flow data for the three freeways.

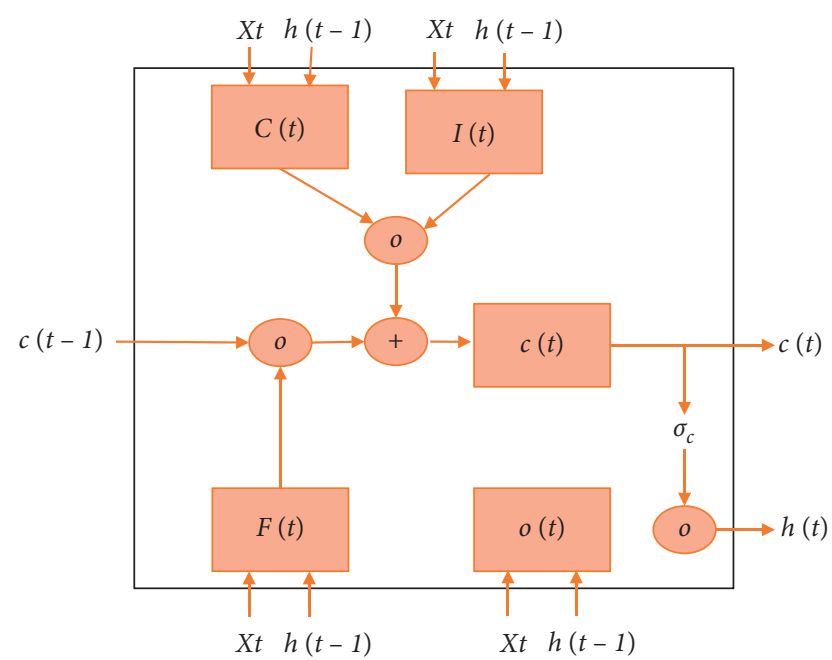

FIGURE 6: LSTM architecture.

where $\sigma_{g}$ is the gate activation function and $W_{i}, W_{f}, W_{c}$, and $W_{o}$ are input weight matrices.

$R_{i}, R_{f}, R_{c}$, and $R_{o}$ are recurrent weight matrices, $X_{t}$ is the input, and $h_{t-1}$ is the output at the previous time $(t-1)$. $b_{i}, b_{f}, b_{c}$, and $b_{o}$ are bias vectors. The forget gate determines how much of the prior memory values should be removed from the cell state. Similarly, the input gate specifies new input to the cell state. Then, the cell state $C_{t}$ and the output $H_{t}$ of the LSTM at time $t$ are calculated as follows:

$$
\begin{aligned}
C_{t} & =f t \odot c t \odot 1+i t \odot g t, \\
H_{t} & =o t \odot \sigma c(c t),
\end{aligned}
$$

where $\odot$ denotes the Hadamard product (element-wise multiplication of vectors).

In this work, the unidirectional and bidirectional LSTM networks were implemented in MATLAB R2020b. First, the data were arranged as two column values: the first column corresponds to speed/flow at time $t$ and the second column corresponds to the expected output $(t+n)$ where $n$ ranges from 5 minutes to 60 minutes into the future. Then, the data were partitioned into training and testing sets. The models were trained on the first $60 \%$ of the sequence and tested on the last $40 \%$. To prevent model overfitting, the training/ testing data were standardized to have zero mean and unit variance. The LSTM networks were created using four layers: sequence input layer (number of features =1), Uni-LSTM/ BiLSTM layers (number of hidden units $=300$ ), fully connected layer (number of responses $=1$ ), and a regression layer. The model hyperparameter settings are presented in Table 1. Multiple sets of hyperparameters were tested with the aim to find the right combination of values which result in the best accuracy. Table 1 shows the parameters that provided the optimal results. The tanh and sigmoid functions were used for state and gate activation functions, 


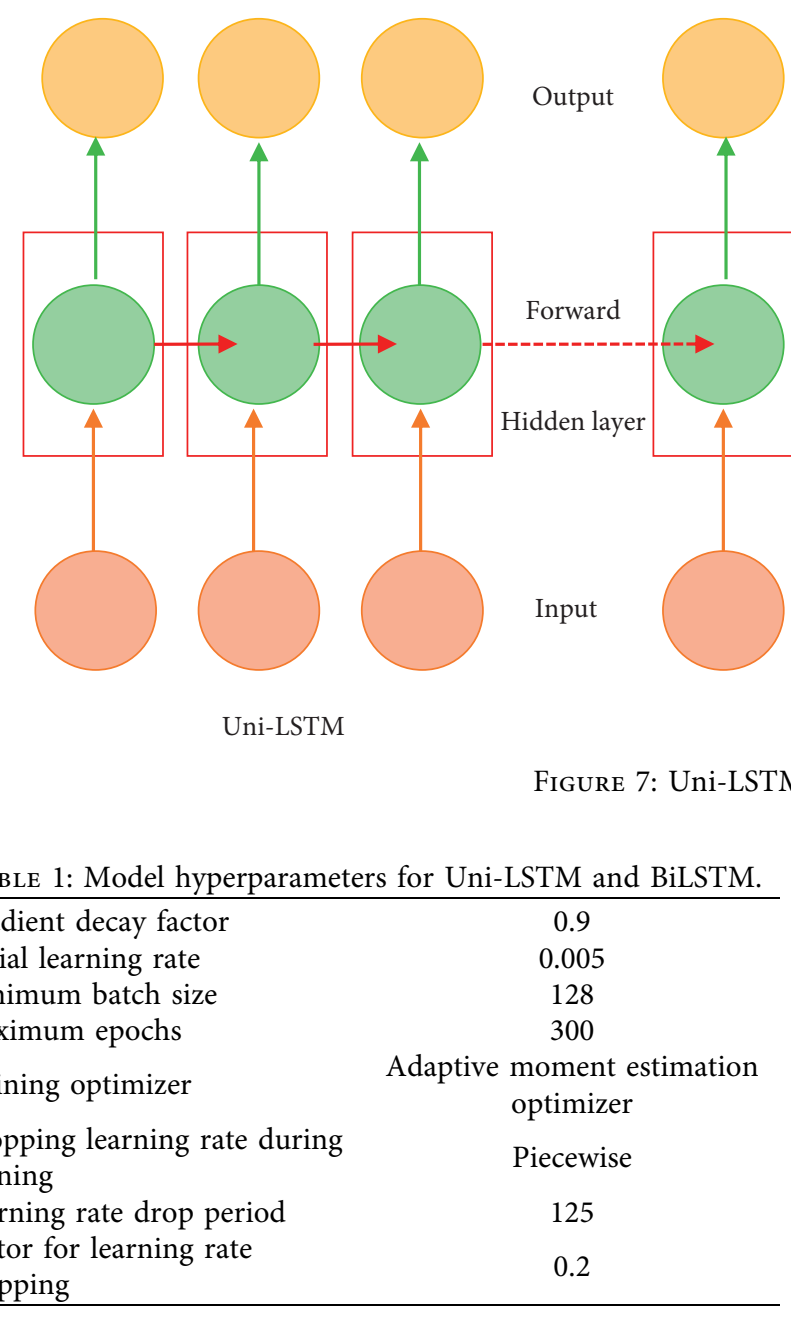

respectively. The LSTM experiments were also implemented in MATLAB R2020b with the Deep Learning Toolbox functions of trainNetwork, training options, and predictAndUpdateState.

\section{Comparative Evaluation of Uni-LSTM and BiLSTM}

The first set of results in this paper was for speed and flow data for the Tullamarine Freeway in Melbourne (Table 2) and the Pacific Motorway in Brisbane (Table 3). The data from both freeways were divided into $60 \%$ training data and $40 \%$ testing data. The mean absolute percentage error (MAPE) is used to calculate the accuracy of the model prediction for different time horizons. MAPE calculates the average absolute difference between the predicted output from the model $(Y 1)$ and expected true output $(Y)$ :

$$
\begin{aligned}
\operatorname{MAPE}(\%) & =\left(\frac{1}{n} \sum_{i=1}^{n} \frac{|Y-Y 1|}{Y}\right) \times 100, \\
\operatorname{accuracy}(\%) & =(100-\mathrm{MAPE}) .
\end{aligned}
$$

The speed prediction results showed that BiLSTM and Uni-LSTM achieve high prediction results up to 60 minutes

\begin{tabular}{lc} 
Gradient decay factor & 0.9 \\
Initial learning rate & 0.005 \\
Minimum batch size & 128 \\
Maximum epochs & 300 \\
Training optimizer & Adaptive moment estimation \\
optimizer \\
tropping learning rate during & Piecewise \\
Learning rate drop period & 125 \\
$\begin{array}{l}\text { Factor for learning rate } \\
\text { dropping }\end{array}$ & 0.2 \\
\hline
\end{tabular}

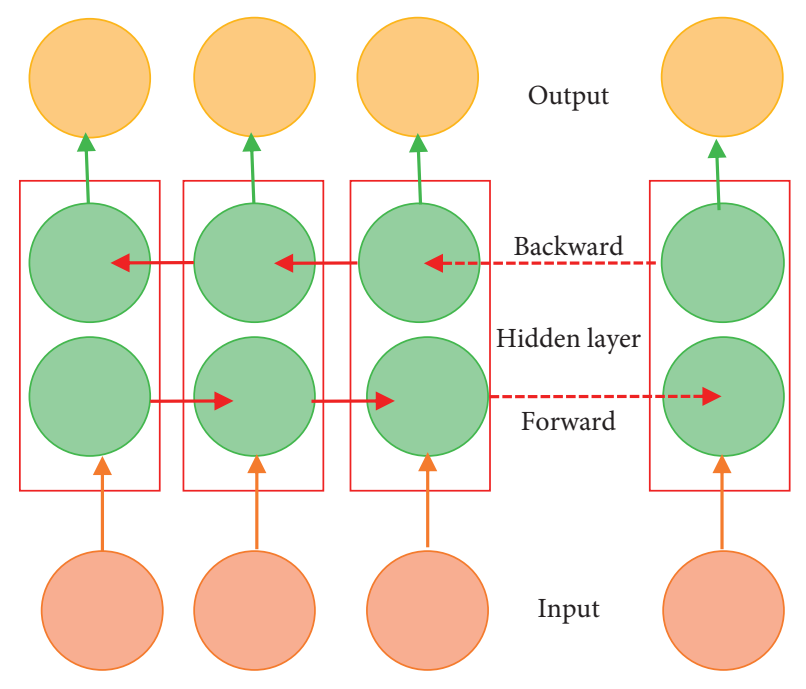

BiLSTM 
TABLE 2: Speed performance for different prediction horizons for the Tullamarine Freeway.

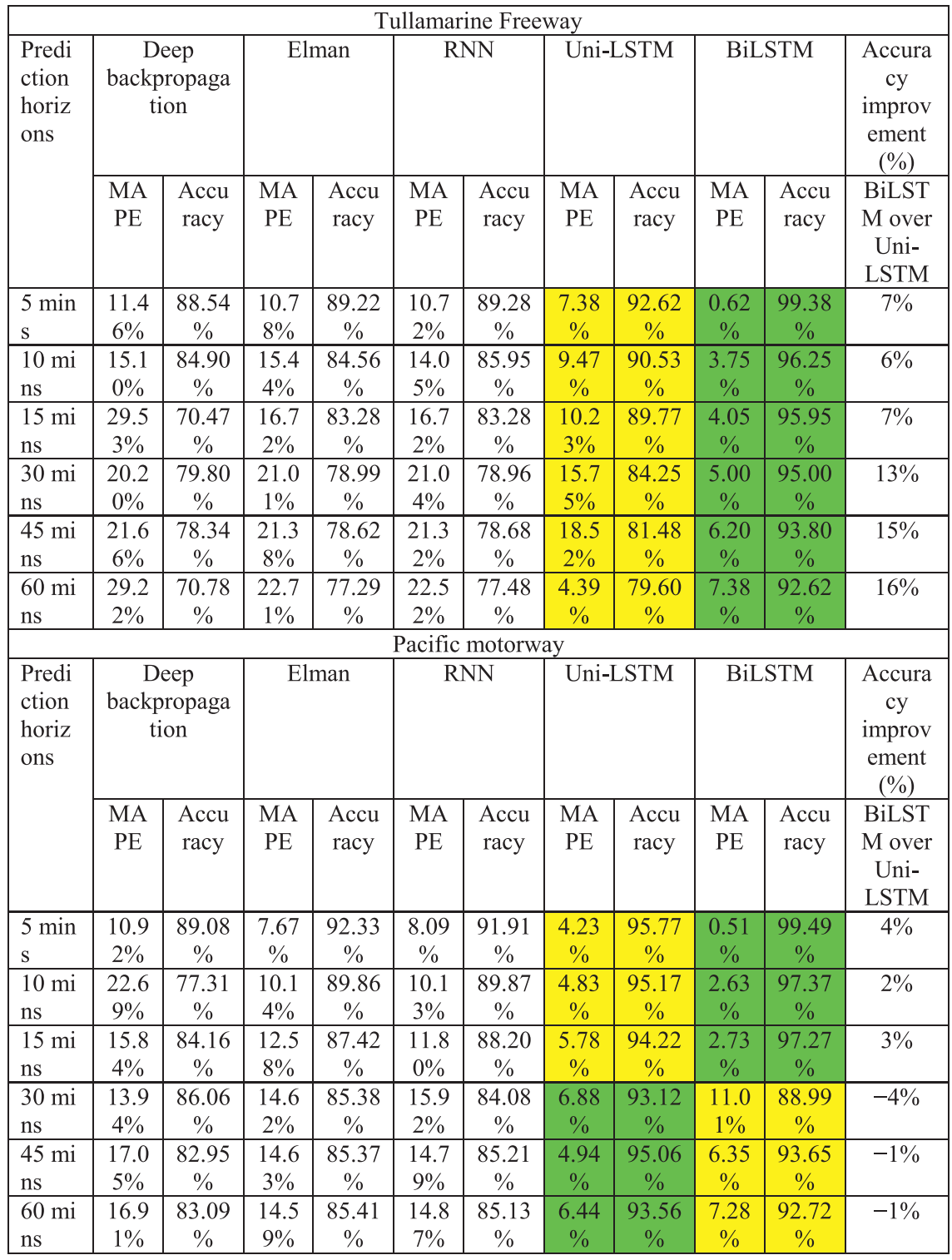

deep Uni-LSTM models for 15-minute prediction horizons on both freeways. For speed, 3-BiLSTM layers and 4BiLSTM layers provide the best accuracy of $98 \%$ on the Tullamarine Freeway, while LSTM layers provide the lowest accuracy of around 94\%. The 4-layered BiLSTM model outperformed other models with $92.5 \%$ accuracy for 15 minute prediction horizons on the Tullamarine Freeway. Similarly, Pacific Motorway experiments show that the 4layer BiLSTM model outperformed other models with an accuracy of $99.99 \%$ as shown in Tables 4 and 5 .

Figures 8 and 9 present the speed and flow results for the 15-minute prediction horizon using the best-performing 4-layered BiLSTM model. These figures compare the target or expected values (blue trendline) with the predicted values from the model (orange trendline). Figure 8 shows the superior performance of the model for predicting both speed and flow with accuracies of $98 \%$ and $92.50 \%$ for 15 -minute prediction horizons on the Tullamarine Freeway. Similarly,
Figure 9 shows a remarkable prediction performance for the 4-layered BiLSTM model on the Pacific Motorway with a prediction accuracy of $99.99 \%$ for both speed and flow for 15-minute prediction horizons.

\section{Deep BiLSTM Model Validation and Transferability}

This section of the paper presents results for model validation and potential for transferability to other freeways without the need for recalibration and retraining. If this can be achieved even at the expense of a depreciated accuracy, it can provide road operators and transport agencies with confidence that they can apply existing models on different freeways even if they have not embarked on comprehensive historical data collection efforts. This also helps them with reducing the cost of deployment of these algorithms by 
TABLE 3: Flow performance for different prediction horizons for the Tullamarine Freeway.

\begin{tabular}{|c|c|c|c|c|c|c|c|c|c|c|c|}
\hline \multicolumn{12}{|c|}{ Tullamarine freeway } \\
\hline \multirow{2}{*}{$\begin{array}{l}\text { Predi } \\
\text { ction } \\
\text { Horiz } \\
\text { ons }\end{array}$} & \multirow{2}{*}{$\begin{array}{c}\begin{array}{c}\text { Deep } \\
\text { Backpro } \\
\text { pagation }\end{array} \\
\text { MAPE }\end{array}$} & \multicolumn{2}{|c|}{ Elman } & \multicolumn{2}{|c|}{ RNN } & \multicolumn{2}{|c|}{ Uni-LSTM } & \multicolumn{2}{|c|}{ BiLSTM } & \multicolumn{2}{|c|}{$\begin{array}{c}\text { Accuracy } \\
\text { Improvemen } \\
t(\%)\end{array}$} \\
\hline & & $\begin{array}{l}\text { Accu } \\
\text { racy }\end{array}$ & $\begin{array}{l}\text { MA } \\
\text { PE }\end{array}$ & $\begin{array}{l}\text { Accu } \\
\text { racy }\end{array}$ & $\begin{array}{c}\text { MA } \\
\text { PE }\end{array}$ & $\begin{array}{c}\text { Accura } \\
\text { cy } \\
\text { Improv } \\
\text { ement } \\
(\%)\end{array}$ & $\begin{array}{l}\text { MA } \\
\text { PE }\end{array}$ & $\begin{array}{l}\text { Accu } \\
\text { racy }\end{array}$ & $\begin{array}{l}\text { MA } \\
\text { PE }\end{array}$ & $\begin{array}{l}\text { Accu } \\
\text { racy }\end{array}$ & $\begin{array}{c}\text { BiL } \\
\text { ST } \\
\text { M } \\
\text { over } \\
\text { Uni- } \\
\text { LST } \\
\text { M }\end{array}$ \\
\hline $\begin{array}{l}5 \min \\
\mathrm{s}\end{array}$ & $29.44 \%$ & $\begin{array}{c}70.5 \\
6 \%\end{array}$ & $\begin{array}{l}25 . \\
45 \\
\%\end{array}$ & $\begin{array}{c}74.5 \\
5 \%\end{array}$ & $\begin{array}{l}25 . \\
35 \\
\%\end{array}$ & $\begin{array}{c}74.65 \\
\%\end{array}$ & $\begin{array}{l}19 . \\
03 \\
\%\end{array}$ & $\begin{array}{c}80.9 \\
7 \%\end{array}$ & $\begin{array}{l}0.3 \\
6 \%\end{array}$ & $\begin{array}{c}99.6 \\
4 \%\end{array}$ & $23 \%$ \\
\hline $\begin{array}{l}10 \mathrm{mi} \\
\mathrm{ns}\end{array}$ & $31.33 \%$ & $\begin{array}{c}68.6 \\
7 \%\end{array}$ & $\begin{array}{l}29 . \\
06 \\
\% \\
\end{array}$ & $\begin{array}{c}70.9 \\
4 \%\end{array}$ & $\begin{array}{l}29 . \\
03 \\
\%\end{array}$ & $\begin{array}{c}70.97 \\
\%\end{array}$ & $\begin{array}{l}23 . \\
15 \\
\%\end{array}$ & $\begin{array}{c}76.8 \\
5 \%\end{array}$ & $\begin{array}{l}9.2 \\
5 \%\end{array}$ & $\begin{array}{c}90.7 \\
5 \%\end{array}$ & $18 \%$ \\
\hline $\begin{array}{l}15 \mathrm{mi} \\
\mathrm{ns}\end{array}$ & $33.73 \%$ & $\begin{array}{c}66.2 \\
7 \%\end{array}$ & $\begin{array}{l}32 . \\
24 \\
\%\end{array}$ & $\begin{array}{c}67.7 \\
6 \%\end{array}$ & $\begin{array}{l}32 . \\
03 \\
\%\end{array}$ & $\begin{array}{c}67.97 \\
\%\end{array}$ & $\begin{array}{l}24 . \\
94 \\
\%\end{array}$ & $\begin{array}{c}75.0 \\
6 \%\end{array}$ & $\begin{array}{l}11 . \\
28 \\
\%\end{array}$ & $\begin{array}{c}88.7 \\
2 \%\end{array}$ & $18 \%$ \\
\hline $\begin{array}{l}30 \mathrm{mi} \\
\mathrm{ns}\end{array}$ & $58.91 \%$ & $\begin{array}{c}41.0 \\
9 \%\end{array}$ & $\begin{array}{l}36 . \\
68 \\
\% \\
\end{array}$ & $\begin{array}{c}63.3 \\
2 \%\end{array}$ & $\begin{array}{l}6 . \\
63 \\
\% \\
\end{array}$ & $\begin{array}{c}63.37 \\
\%\end{array}$ & $\begin{array}{l}35 . \\
95 \\
\% \\
\end{array}$ & $\begin{array}{c}64.0 \\
5 \%\end{array}$ & $\begin{array}{l}14 . \\
10 \\
\% \\
\end{array}$ & $\begin{array}{c}85.9 \\
0 \%\end{array}$ & $34 \%$ \\
\hline $\begin{array}{l}45 \mathrm{mi} \\
\mathrm{ns}\end{array}$ & $41.53 \%$ & $\begin{array}{c}58.4 \\
7 \%\end{array}$ & $\begin{array}{l}38 . \\
21 \\
\%\end{array}$ & $\begin{array}{c}61.7 \\
9 \%\end{array}$ & $\begin{array}{l}38 . \\
27 \\
\%\end{array}$ & $\begin{array}{c}61.73 \\
\%\end{array}$ & $\begin{array}{l}37 . \\
80 \\
\%\end{array}$ & $\begin{array}{c}62.2 \\
0 \%\end{array}$ & $\begin{array}{l}16 . \\
11 \\
\%\end{array}$ & $\begin{array}{c}83.8 \\
9 \%\end{array}$ & $35 \%$ \\
\hline $\begin{array}{l}60 \mathrm{mi} \\
\mathrm{ns}\end{array}$ & $43.69 \%$ & $\begin{array}{c}56.3 \\
1 \%\end{array}$ & $\begin{array}{c}39 . \\
00 \\
\% \\
\end{array}$ & $\begin{array}{c}61.0 \\
0 \%\end{array}$ & $\begin{array}{l}39 . \\
14 \\
\% \\
\end{array}$ & $\begin{array}{c}60.86 \\
\%\end{array}$ & $\begin{array}{l}37 . \\
02 \\
\% \\
\end{array}$ & $\begin{array}{c}62.9 \\
8 \%\end{array}$ & $\begin{array}{l}25 . \\
14 \\
\% \\
\end{array}$ & $\begin{array}{c}74.8 \\
6 \%\end{array}$ & $19 \%$ \\
\hline \multicolumn{12}{|c|}{ Pacific motorway } \\
\hline \multirow{2}{*}{$\begin{array}{l}\text { Predi } \\
\text { ction } \\
\text { Horiz } \\
\text { ons }\end{array}$} & $\begin{array}{c}\text { Deep } \\
\text { Backpro } \\
\text { pagation }\end{array}$ & \multicolumn{2}{|c|}{ Elman } & \multicolumn{2}{|c|}{ RNN } & \multicolumn{2}{|c|}{ Uni-LSTM } & \multicolumn{2}{|c|}{ BiLSTM } & \multicolumn{2}{|c|}{$\begin{array}{c}\text { Accuracy } \\
\text { Improvemen } \\
t(\%)\end{array}$} \\
\hline & MAPE & $\begin{array}{l}\text { Accu } \\
\text { racy }\end{array}$ & $\begin{array}{l}\text { MA } \\
\text { PE }\end{array}$ & $\begin{array}{l}\text { Accu } \\
\text { racy }\end{array}$ & $\begin{array}{c}\text { MA } \\
\text { PE }\end{array}$ & $\begin{array}{c}\text { Accura } \\
\text { cy } \\
\text { Improv } \\
\text { ement } \\
(\%)\end{array}$ & $\begin{array}{l}\text { MA } \\
\text { PE }\end{array}$ & $\begin{array}{l}\text { Accu } \\
\text { racy }\end{array}$ & $\begin{array}{l}\text { MA } \\
\text { PE }\end{array}$ & $\begin{array}{c}\text { Accu } \\
\text { racy }\end{array}$ & $\begin{array}{c}\text { BiL } \\
\text { ST } \\
\text { M } \\
\text { over } \\
\text { Uni- } \\
\text { LST } \\
\text { M }\end{array}$ \\
\hline $\begin{array}{l}5 \min \\
\mathrm{s}\end{array}$ & $27.24 \%$ & $\begin{array}{c}72.7 \\
6 \%\end{array}$ & $\begin{array}{l}21 . \\
10 \\
\%\end{array}$ & $\begin{array}{c}78.9 \\
0 \%\end{array}$ & $\begin{array}{l}21 . \\
02 \\
\%\end{array}$ & $\begin{array}{c}78.98 \\
\%\end{array}$ & $\begin{array}{l}13 . \\
07 \\
\%\end{array}$ & $\begin{array}{c}86.9 \\
3 \%\end{array}$ & $\begin{array}{l}0.5 \\
0 \%\end{array}$ & $\begin{array}{c}99.5 \\
0 \%\end{array}$ & $14 \%$ \\
\hline $\begin{array}{l}10 \mathrm{mi} \\
\mathrm{ns}\end{array}$ & $22.51 \%$ & $\begin{array}{c}77.4 \\
9 \%\end{array}$ & $\begin{array}{l}22 . \\
21 \\
\% \\
\end{array}$ & $\begin{array}{c}77.7 \\
9 \%\end{array}$ & $\begin{array}{l}21 . \\
63 \\
\% \\
\end{array}$ & $\begin{array}{c}78.37 \\
\%\end{array}$ & $\begin{array}{l}17 . \\
14 \\
\%\end{array}$ & $\begin{array}{c}82.8 \\
6 \%\end{array}$ & $\begin{array}{l}5.7 \\
5 \%\end{array}$ & $\begin{array}{c}94.2 \\
5 \%\end{array}$ & $14 \%$ \\
\hline $\begin{array}{l}15 \mathrm{mi} \\
\mathrm{ns}\end{array}$ & $26.62 \%$ & $\begin{array}{c}73.3 \\
8 \%\end{array}$ & $\begin{array}{l}22 . \\
10 \\
\%\end{array}$ & $\begin{array}{c}77.9 \\
0 \%\end{array}$ & $\begin{array}{l}22 . \\
09 \\
\%\end{array}$ & $\begin{array}{c}77.91 \\
\%\end{array}$ & $\begin{array}{l}14 . \\
39 \\
\%\end{array}$ & $\begin{array}{c}85.6 \\
1 \%\end{array}$ & $\begin{array}{l}6.7 \\
7 \%\end{array}$ & $\begin{array}{c}93.2 \\
3 \%\end{array}$ & $9 \%$ \\
\hline $\begin{array}{l}30 \mathrm{mi} \\
\mathrm{ns}\end{array}$ & $24.21 \%$ & $\begin{array}{c}75.7 \\
9 \%\end{array}$ & $\begin{array}{l}24 . \\
72 \\
\%\end{array}$ & $\begin{array}{c}75.2 \\
8 \%\end{array}$ & $\begin{array}{l}24 . \\
72 \\
\%\end{array}$ & $\begin{array}{c}75.28 \\
\%\end{array}$ & $\begin{array}{l}18 . \\
23 \\
\%\end{array}$ & $\begin{array}{c}81.7 \\
7 \%\end{array}$ & $\begin{array}{l}11 . \\
35 \\
\%\end{array}$ & $\begin{array}{c}88.6 \\
5 \%\end{array}$ & $8 \%$ \\
\hline $\begin{array}{l}45 \mathrm{mi} \\
\mathrm{ns}\end{array}$ & $30.95 \%$ & $\begin{array}{c}69.0 \\
5 \%\end{array}$ & $\begin{array}{l}26 . \\
66 \\
\%\end{array}$ & $\begin{array}{c}73.3 \\
4 \%\end{array}$ & $\begin{array}{l}26 . \\
98 \\
\%\end{array}$ & $\begin{array}{c}73.02 \\
\%\end{array}$ & $\begin{array}{l}16 . \\
39 \\
\%\end{array}$ & $\begin{array}{c}83.6 \\
1 \%\end{array}$ & $\begin{array}{l}14 . \\
34 \\
\%\end{array}$ & $\begin{array}{c}85.6 \\
6 \%\end{array}$ & $2 \%$ \\
\hline $\begin{array}{l}60 \mathrm{mi} \\
\mathrm{ns}\end{array}$ & $26.92 \%$ & $\begin{array}{c}73.0 \\
8 \%\end{array}$ & $\begin{array}{l}27 . \\
64 \\
\%\end{array}$ & $\begin{array}{c}72.3 \\
6 \%\end{array}$ & $\begin{array}{l}27 . \\
49 \\
\%\end{array}$ & $\begin{array}{c}72.51 \\
\%\end{array}$ & $\begin{array}{l}15 . \\
89 \\
\%\end{array}$ & $\begin{array}{c}84.1 \\
1 \%\end{array}$ & $\begin{array}{l}15 . \\
90 \\
\%\end{array}$ & $\begin{array}{c}84.1 \\
0 \%\end{array}$ & $0 \%$ \\
\hline
\end{tabular}

avoiding the need to preprocess new data and calibrate and validate new models which is a time-consuming undertaking that requires substantial resources and experienced and welltrained AI staff and specialists.
The model validation experimental design is shown in Figure 10. The learning obtained from the previous comparative evaluations was used to develop robust speed and flow prediction models using data combined from the two 
TABLE 4: Speed and flow performance for different prediction horizons for the Tullamarine Freeway.

\begin{tabular}{|l|c|c|c|c|}
\hline \multicolumn{4}{|c|}{ Tullamarine freeway $(15$ min prediction horizon) } \\
\hline \multirow{2}{*}{$\begin{array}{l}\text { Model architecture and number of } \\
\text { layers }\end{array}$} & \multicolumn{2}{|c|}{ Speed } & \multicolumn{2}{c|}{ Flow } \\
\cline { 2 - 5 } 1 LSTM layer & MAPE $\%$ & Accuracy $\%$ & MAPE $\%$ & Accuracy \% \\
\hline 2 LSTM layers & $10.23 \%$ & $89.77 \%$ & $24.94 \%$ & $75.06 \%$ \\
\hline 3 LSTM layers & $8.13 \%$ & $91.87 \%$ & $28.09 \%$ & $71.92 \%$ \\
\hline 4 LSTM layers & $6.03 \%$ & $90.97 \%$ & $25.74 \%$ & $74.26 \%$ \\
\hline 1 BiLSTM layer & $4.05 \%$ & $93.80 \%$ & $13.75 \%$ & $86.25 \%$ \\
\hline 2 BiLSTM layers & $2.51 \%$ & $97.49 \%$ & $11.28 \%$ & $88.72 \%$ \\
\hline 3 BiLSTM layers & $2.00 \%$ & $98.00 \%$ & $8.67 \%$ & $91.94 \%$ \\
\hline 4 BiLSTM layers & $2.00 \%$ & $98.00 \%$ & $7.49 \%$ & $92.33 \%$ \\
\hline 1 BiLSTM layer, 1 LSTM & $2.98 \%$ & $97.02 \%$ & $8.05 \%$ & $91.95 \%$ \\
\hline 2 BiLSTM layers, 1 LSTM & $2.25 \%$ & $97.75 \%$ & $10.65 \%$ & $89.35 \%$ \\
\hline 3 BiLSTM layers, 1 LSTM & $2.25 \%$ & $97.75 \%$ & $12.84 \%$ & $87.16 \%$ \\
\hline
\end{tabular}

TABLE 5: Speed and flow performance for different prediction horizons for the Pacific Motorway.

\begin{tabular}{|l|c|c|c|c|}
\hline \multirow{4}{*}{ Pacific motorway (15 min interval) } \\
\cline { 2 - 5 } $\begin{array}{l}\text { Model architecture and number of } \\
\text { layers }\end{array}$ & $\begin{array}{c}|c| \\
\text { MAPE } \\
\%\end{array}$ & Accuracy $\%$ & MAPE $\%$ & Accuracy $\%$ \\
\hline 1 LSTM layer & $5.78 \%$ & $94.22 \%$ & $14.39 \%$ & $85.61 \%$ \\
\hline 2 LSTM layers & $4.07 \%$ & $95.93 \%$ & $0.97 \%$ & $99.04 \%$ \\
\hline 3 LSTM layers & $3.51 \%$ & $96.49 \%$ & $3.36 \%$ & $96.64 \%$ \\
\hline 4 LSTM layers & $6.12 \%$ & $93.88 \%$ & $0.53 \%$ & $99.47 \%$ \\
\hline 1 BiLSTM layer & $2.73 \%$ & $97.27 \%$ & $6.77 \%$ & $93.23 \%$ \\
\hline 2 BiLSTM layers & $0.65 \%$ & $99.35 \%$ & $0.12 \%$ & $99.88 \%$ \\
\hline 3 BiLSTM layers & $0.02 \%$ & $99.98 \%$ & $0.10 \%$ & $99.89 \%$ \\
\hline 4 BiLSTM layers & $0.01 \%$ & $99.99 \%$ & $0.01 \%$ & $99.99 \%$ \\
\hline 1 BiLSTM layer, 1 LSTM & $0.91 \%$ & $99.09 \%$ & $0.14 \%$ & $99.86 \%$ \\
\hline 2 BiLSTM layers, 1 LSTM & $0.60 \%$ & $99.39 \%$ & $0.02 \%$ & $99.98 \%$ \\
\hline 3 BiLSTM layers, 1 LSTM & $0.01 \%$ & $99.99 \%$ & $0.02 \%$ & $99.98 \%$ \\
\hline
\end{tabular}

largest datasets (Tullamarine and South Eastern Freeways in Melbourne). The data used in model development included 24,270 observations and were divided into two sets: training set comprising $60 \%$ of the data (14,562 observations) and testing set comprising $40 \%$ of the data ( 9,708 observations). The validation dataset included 1,667 observations from the third freeway (Pacific Motorway in Brisbane). The model development results are provided in the first set of columns in Table 6 (Tullamarine and South Eastern Freeways). For speed, the model accuracy ranged from $99.7 \%$ for 5 -minute forecasting horizons to $91.8 \%$ for 60 -minute forecasting horizons. For flow, the model accuracy ranged from $99.6 \%$ for 5 -minute forecasting horizons to $71.2 \%$ for 60 -minute forecasting horizons. The model was then validated (without retraining) on the third independent dataset from the Pacific Motorway in Brisbane.
The validation results are shown in the right side columns of Table 6. For speed, the model's accuracy ranged from $99.7 \%$ for 5 -minute forecasting horizons to $90.2 \%$ for 60-minute forecasting horizons. For traffic flow, the model's accuracy ranged from $97.2 \%$ for 5 -minute forecasting horizons to $82.1 \%$ for 30 -minute forecasting horizons. The performance degrades to $79.19 \%$ for 45 -minute and $73.45 \%$ for 60-minute prediction horizons, as shown in Table 6. These findings are also depicted in Figures 11-16.

In Figures 11-16, the blue trendline represents the targeted real data compared to the orange trendline which represents the results generated from the model. In Figure 13, the difference between targeted and predicted results for both speed and flow is minimal as the MAPE percentages between the two are $0.27 \%$ and $2.83 \%$, respectively. In Figure 14, the speed results for 10 -minute prediction 


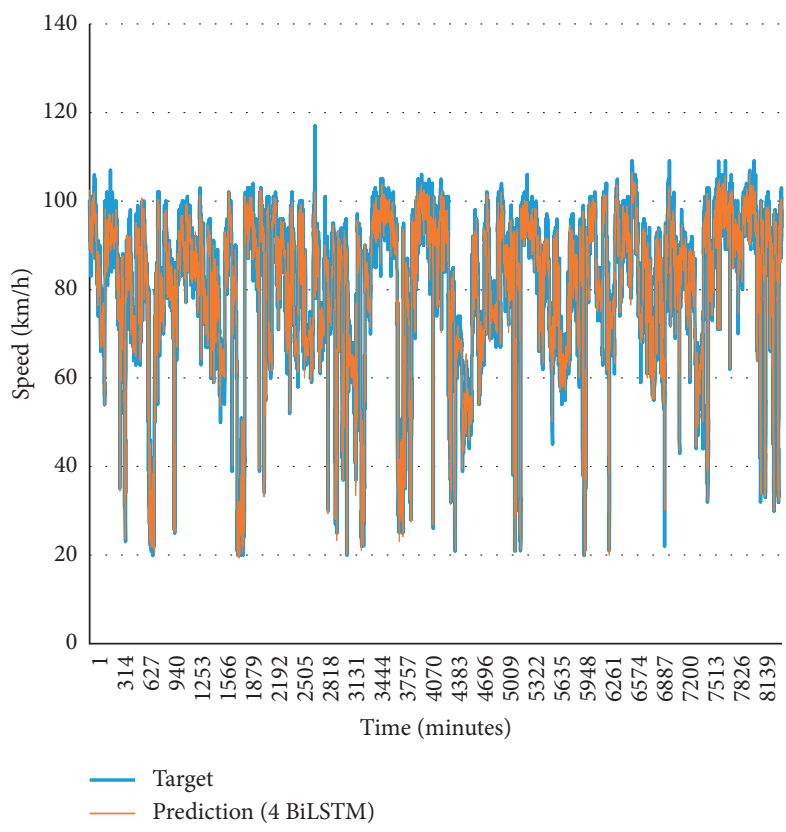

(a)

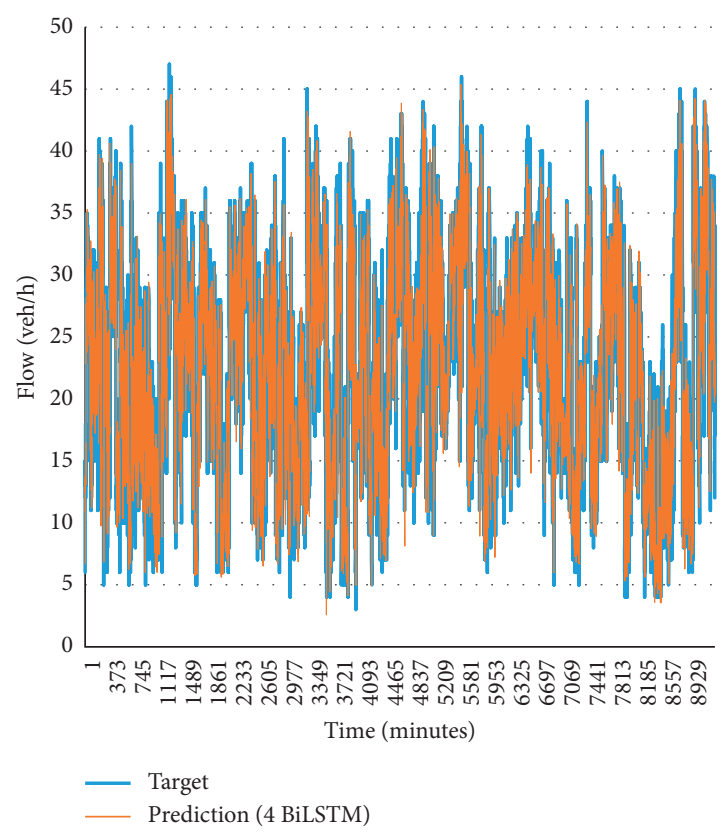

(b)

Figure 8: Tullamarine Freeway speed and flow prediction results for 15-minute horizons using the 4-BiLSTM model.

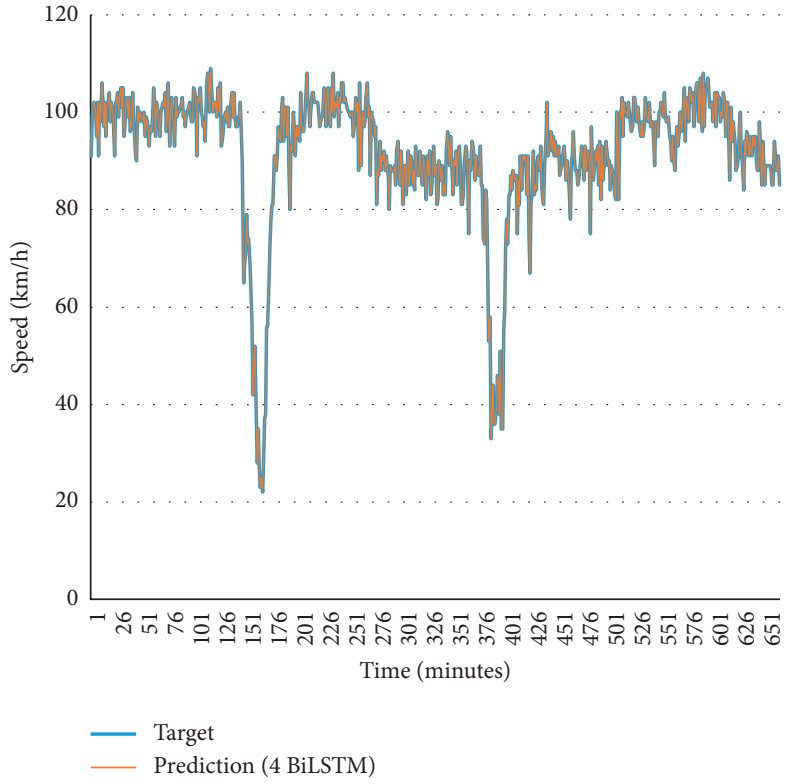

(a)

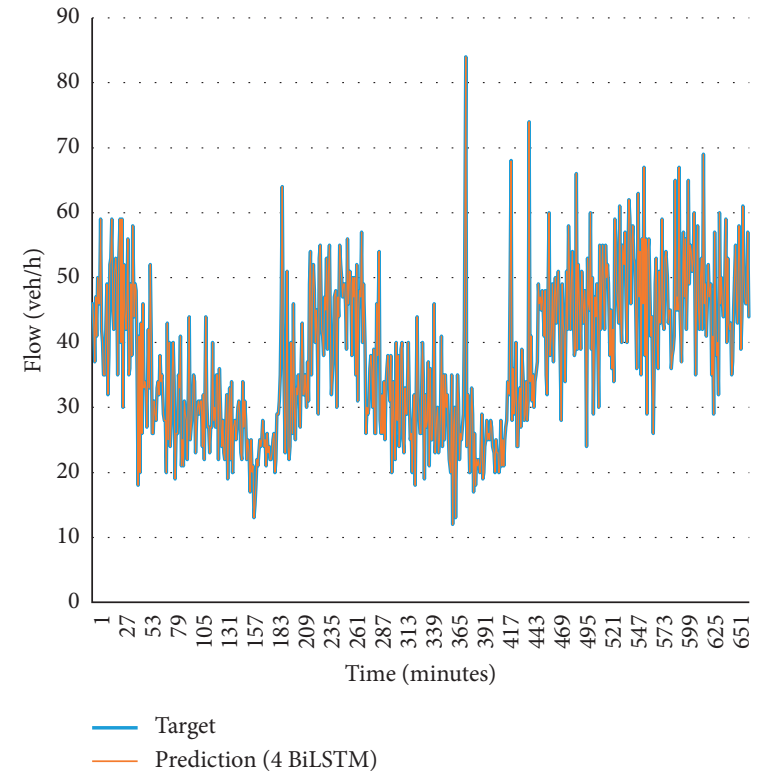

(b)

Figure 9: Pacific Motorway speed and flow prediction results for 15-minute horizons using the 4-BiLSTM model.

horizons also demonstrate a low MAPE percentage error of $3.02 \%$ compared to $9.26 \%$ for flow. As expected, it can be noted that the error increases as the prediction horizon increases. In Figure 15, the MAPE percentage error increases minimally for 15-minute prediction horizons for speed (4.63\% increase) compared to a high increase in error for flow (17.49\%). The same behaviour is observed for 30- minute prediction horizons as the errors for speed and flow increase to $6.28 \%$ and $17.91 \%$ as shown in Figure 16. These results suggest that the model is able to accurately validate speed to multiple prediction horizons. The flow prediction results also showed good accuracies higher than $80 \%$ for 5 , 10,15 , and 30 minutes into the future using the 4-layered BiLSTM model. 


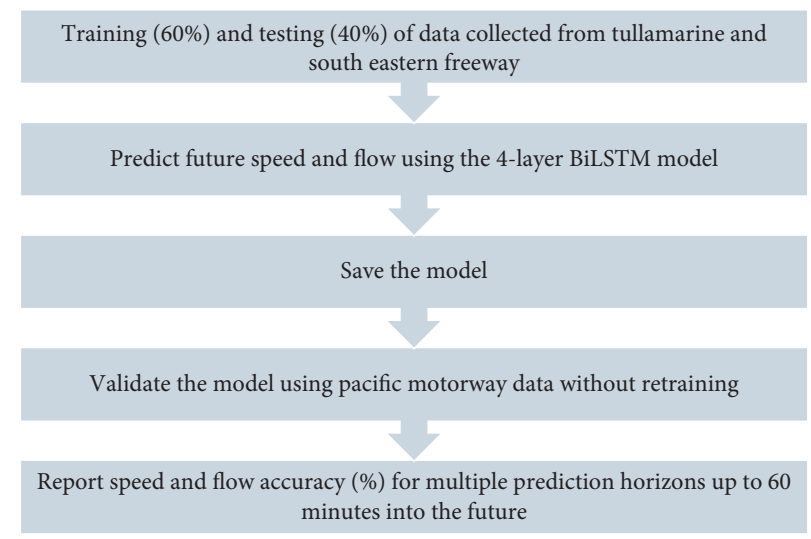

FIgURE 10: Validation experiment design.

TABLE 6: Speed and flow validation results for different prediction horizons.

\begin{tabular}{|c|c|c|c|c|c|c|c|c|}
\hline \multirow{3}{*}{ Prediction horizons } & \multicolumn{4}{|c|}{ Tullamarine Freeway + South Eastern Freeway } & \multicolumn{4}{|c|}{ Validation on the Pacific Motorway } \\
\hline & \multicolumn{2}{|c|}{ Speed $(\mathrm{km} / \mathrm{h})$} & \multicolumn{2}{|c|}{ Flow (veh/h) } & \multicolumn{2}{|c|}{ Speed $(\mathrm{km} / \mathrm{h})$} & \multicolumn{2}{|c|}{ Flow (veh/h) } \\
\hline & MAPE (\%) & Accuracy $(\%)$ & MAPE (\%) & Accuracy (\%) & MAPE (\%) & Accuracy $(\%)$ & MAPE (\%) & Accuracy (\%) \\
\hline $5 \mathrm{mins}$ & 0.30 & 99.70 & 0.38 & 99.62 & 0.27 & 99.73 & 2.83 & 97.17 \\
\hline 10 mins & 2.61 & 97.39 & 4.15 & 95.85 & 3.02 & 96.98 & 9.26 & 90.74 \\
\hline 15 mins & 3.06 & 96.94 & 6.82 & 93.18 & 4.63 & 95.37 & 17.49 & 82.51 \\
\hline 30 mins & 8.14 & 91.86 & 11.26 & 88.74 & 6.28 & 93.72 & 17.91 & 82.09 \\
\hline $45 \mathrm{mins}$ & 7.74 & 92.26 & 19.01 & 80.99 & 7.84 & 92.16 & 20.81 & 79.19 \\
\hline $60 \mathrm{mins}$ & 8.17 & 91.83 & 28.77 & 71.23 & 9.83 & 90.17 & 26.55 & 73.45 \\
\hline
\end{tabular}

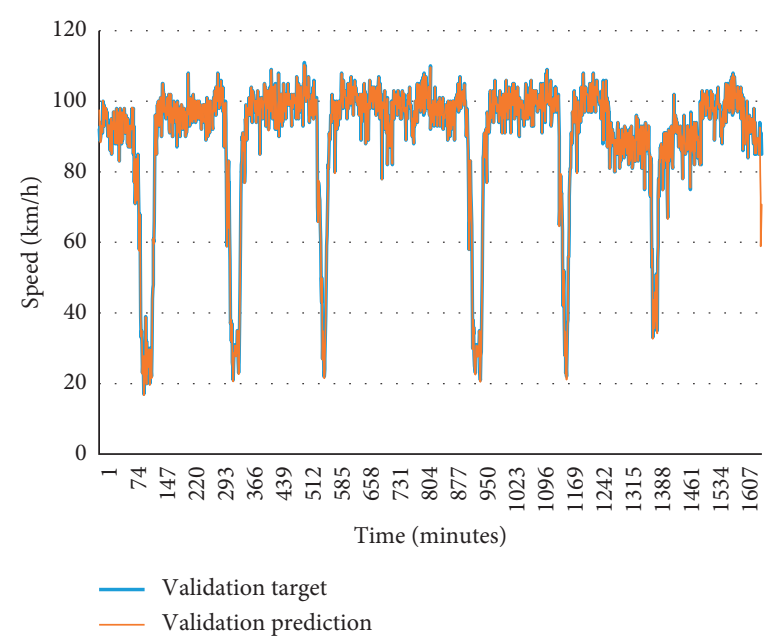

(a)

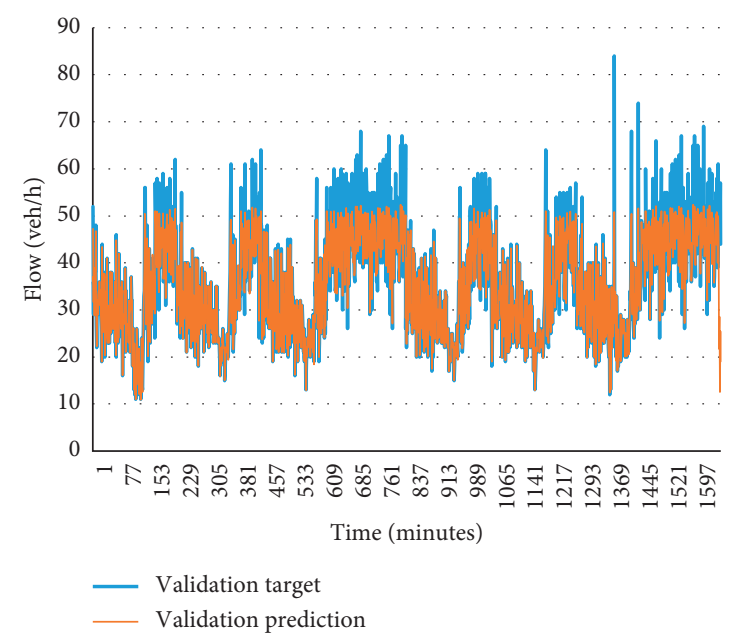

(b)

FIgURE 11: Speed and flow validation results for 5 minutes. 


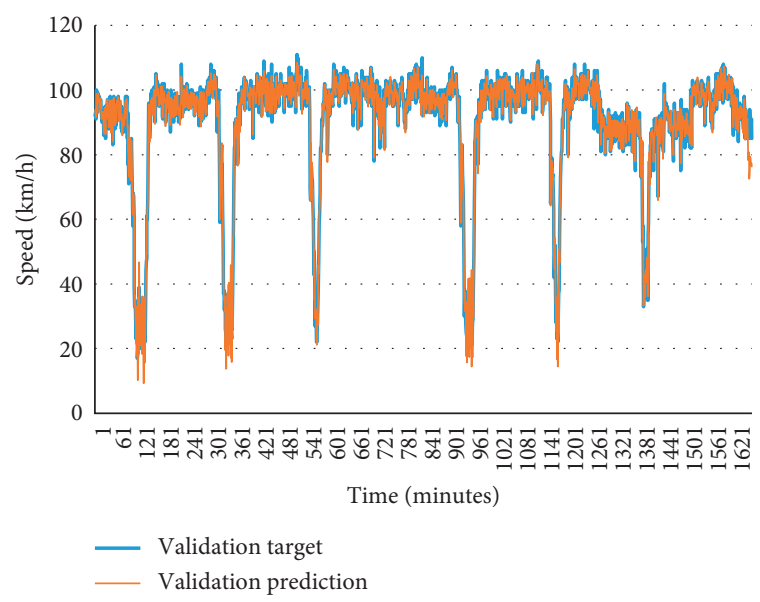

(a)

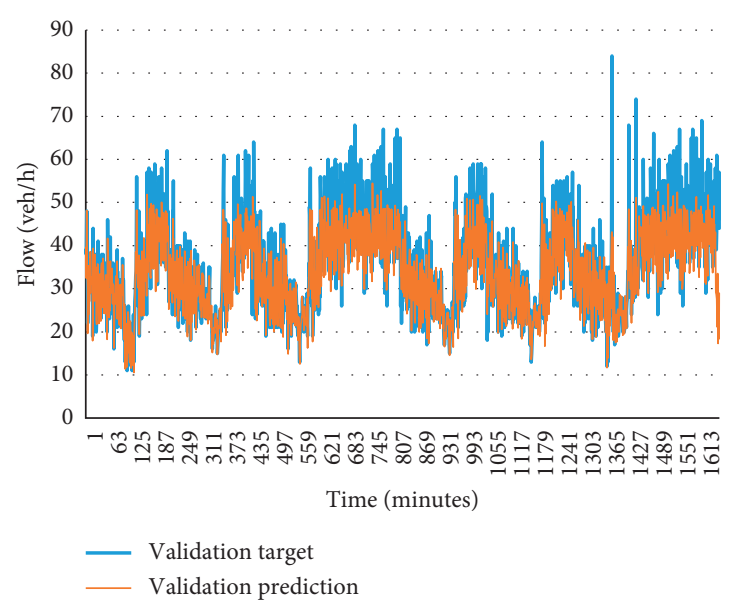

(b)

Figure 12: Speed and flow validation results for 10 minutes.

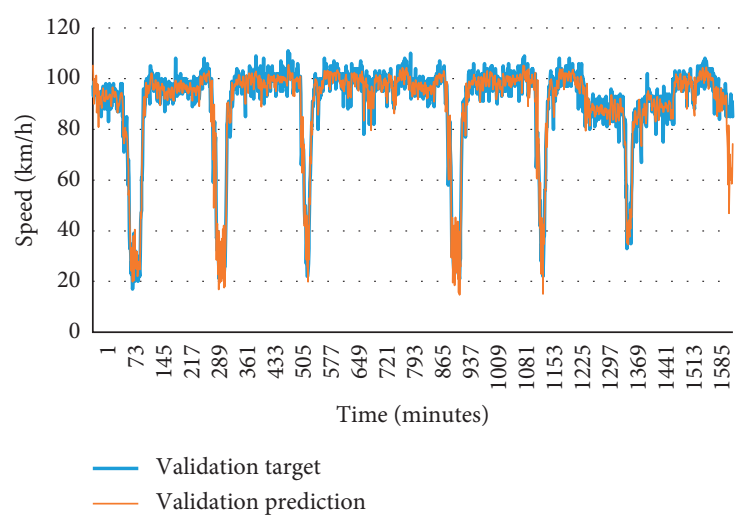

(a)

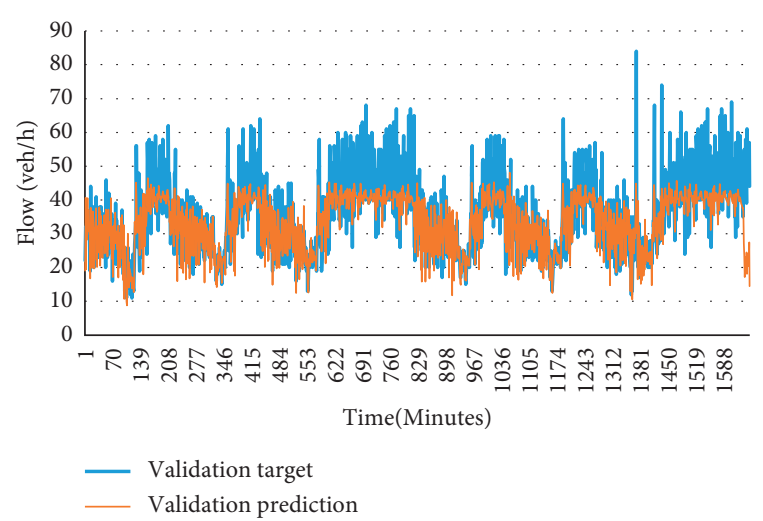

(b)

FIGURE 13: Speed and flow validation results for 15 minutes.

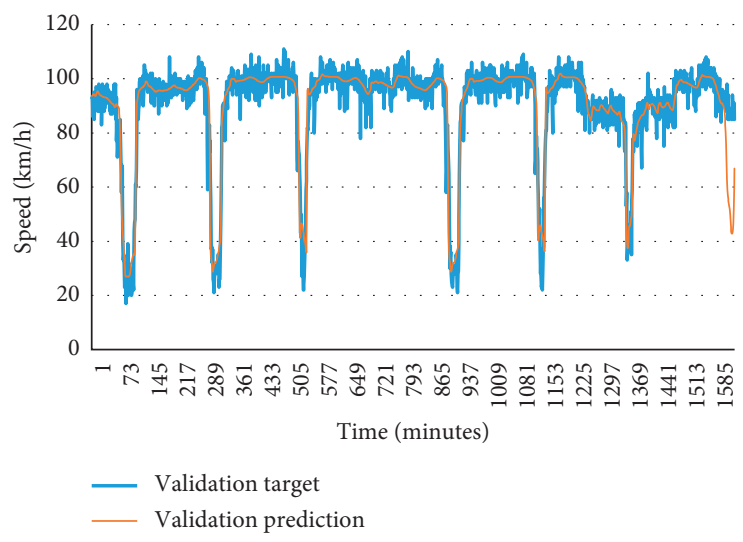

(a)

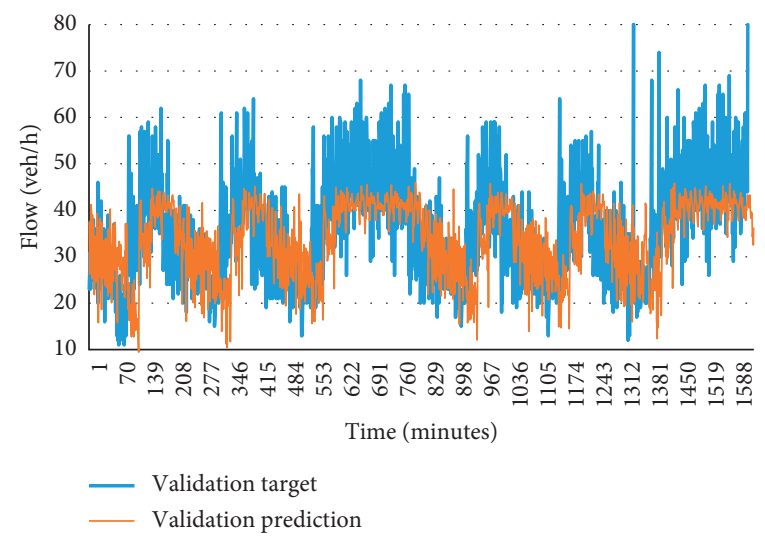

(b)

Figure 14: Speed and flow validation results for 30 minutes. 


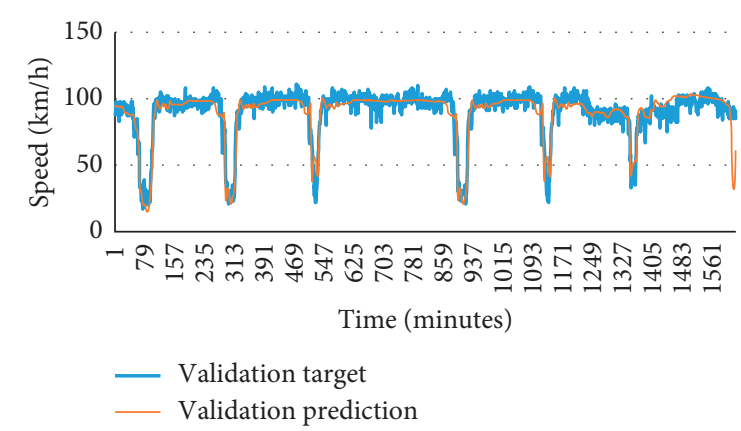

(a)

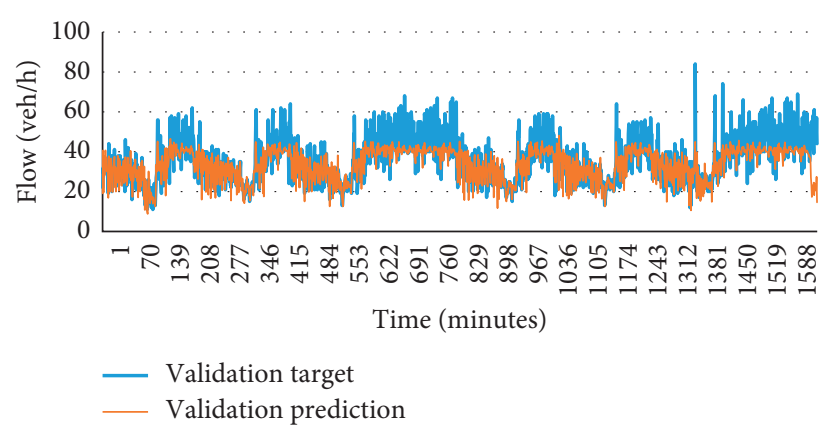

(b)

FIGURE 15: Speed and flow validation results for 45 minutes.

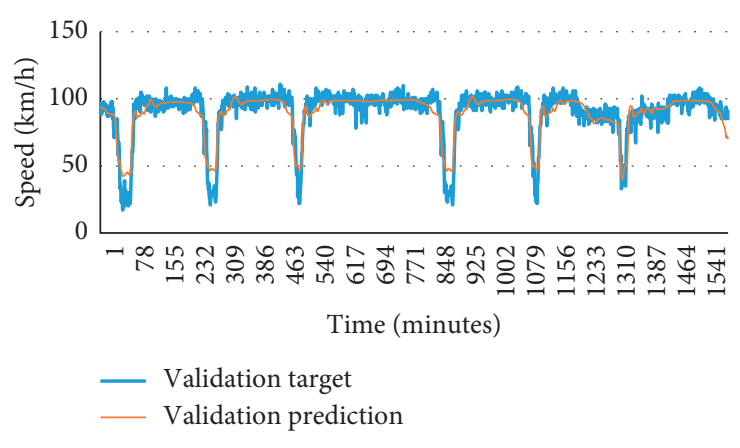

(a)

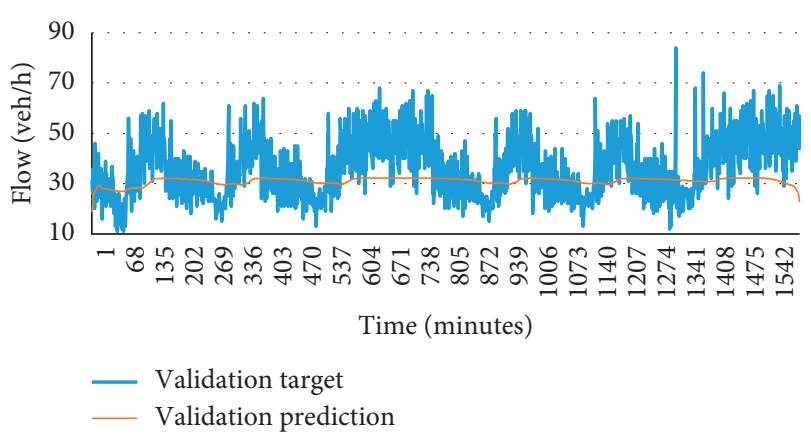

(b)

FIGURE 16: Speed and flow validation results for 60 minutes.

\section{Conclusions, Contributions, and Future Research Directions}

In this paper, unidirectional and bidirectional LSTM networks were developed to predict speed and flow on freeways for forecasting horizons up to 60 minutes into the future. The models were evaluated based on historical field data collected from inductive loop sensors on a number of freeways in Australia. A comprehensive and rigorous procedure was adopted to evaluate the suitability of different architectures and modelling parameters. The results showed a superior performance for the bidirectional compared to unidirectional LSTM. The results also demonstrated the challenges of predicting traffic flow, compared to speed. This was a result of the noisy nature of flow measurements compared to speed observations. For the Tullamarine Freeway, the BiLSTM model was able to achieve speed predictions up to 60 minutes into the future with an accuracy above $90 \%$. For the flow prediction, the accuracy was above $80 \%$ up to $45 \mathrm{~min}$ forecasting horizons, outperforming the Uni-LSTM model. For the Pacific Motorway, BiLSTM also outperformed Uni-LSTM with accuracies above $88 \%$ for speed and above $80 \%$ for flow up to 60 -minute prediction horizons.

This study also extended the models and evaluated their performance when adding multiple Uni-LSTM and BiLSTM layers or mixing both LSTM and BiLSTM as one model for 15-minute prediction horizons. The experiments showed that the 4-layered BiLSTM outperformed other models for both speed and flow on Tullamarine and Pacific Motorway datasets. Another contribution of this work was to examine model validation and its potential for transferability. The evaluation was undertaken on a combined dataset from the Tullamarine and South Eastern Freeways in Melbourne. This approach enabled us to train the models on a large dataset with different patterns and variable traffic conditions, including peak, nonpeak, weekday, weekend, and incident data. Once optimized, the model was validated by testing only (without retraining) on an independent dataset from a third freeway. The validation results showed speed prediction accuracies ranging from $99.7 \%$ for 5 -minute forecasting horizons to $90.2 \%$ for 60 -minute forecasting horizons. The flow validation prediction accuracies were lower and ranged between $74 \%$ and $97 \%$. While it is acknowledged that more comprehensive testing is required on much larger numbers of freeways, this contribution demonstrates the potential to develop transferable models provided sufficient data are available to represent more diverse traffic conditions from different cities around the world.

Future directions in this research include collection of more field data from other real-life freeways in different cities both in Australia and overseas such as those reported in [44]. The use of microsimulation to generate edge case data that are difficult to measure in the field is also 
recommended $[45,46]$. As was shown in this paper, training the models on different patterns and variable traffic conditions enabled us to develop robust models that can perform well on an independent dataset. With more data used for training and model development, it is expected that the accuracy will improve. The AI field is also witnessing a fast pace of developments and breakthroughs providing future opportunities to test new architectures to further improve the model performance and accuracy.

\section{Data Availability}

To ensure transparency and allow other researchers to audit and reproduce the results reported in this study, the field data used in this work can be found at https://bit.ly/2Ka0RiS.

\section{Conflicts of Interest}

The authors declare no conflicts of interest.

\section{Acknowledgments}

The first author would like to acknowledge her Ph.D. scholarship provided by the Iraqi Government and Swinburne University of Technology in Melbourne, Australia.

\section{References}

[1] H. Dia, D. Harney, and A. Boyle, "Dynamics of drivers' route choice decisions under advanced traveller information systems," Road \& Transport Research, vol. 10, no. 4, p. 3, 2001.

[2] R. Abduljabbar and H. DIA, "Predictive intelligence: a neural network learning system for traffic condition prediction and monitoring on freeways," Journal of the Eastern Asia Society for Transportation Studies, vol. 13, pp. 1785-1800, 2019.

[3] R. Abduljabbar and H. Dia, "A deep learning approach for freeway vehicle speed and flow prediction," in Proceedings of the Australasian Transport Research Forum (ATRF), 41st, 2019, Canberra, ACT, Australia, October 2019.

[4] K. Thomas, H. Dia, and N. Cottman, "January. Simulation of arterial incident detection using neural networks," in Proceedings of the 8th World Congress on ITS, Sydney, Australia, October 2001.

[5] S. Nigarnjanagool and H. Dia, "Evaluation of a dynamic signal optimisation control model using traffic simulation," IATSS Research, vol. 29, no. 1, pp. 22-30, 2005.

[6] K. Thomas and H. Dia, "Comparative evaluation of freeway incident detection models using field data," IEE ProceedingsIntelligent Transport Systems, vol. 153, no. 3, pp. 230-241, 2006.

[7] J. Tang, F. Liu, Y. Zou, W. Zhang, and Y. Wang, "An improved fuzzy neural network for traffic speed prediction considering periodic characteristic," IEEE Transactions on Intelligent Transportation Systems, vol. 18, no. 9, pp. 2340-2350, 2017.

[8] J. Tang, X. Chen, Z. Hu, F. Zong, C. Han, and L. Li, "Traffic flow prediction based on combination of support vector machine and data denoising schemes," Physica A: Statistical Mechanics and Its Applications, vol. 534, Article ID 120642, 2019.

[9] H. Dia, G. Rose, and A. Snell, "Comparative performance of freeway automated incident detection algorithms," in Institute of Transport and Logistics Studies Working Paper ITS-WP-96-
15The University of Sydney, Sydney, Australia, 1996, https:// ses.library.usyd.edu.au/handle/2123/19426.

[10] X. Ma, Z. Tao, Y. Wang, H. Yu, and Y. Wang, "Long shortterm memory neural network for traffic speed prediction using remote microwave sensor data," Transportation Research Part C: Emerging Technologies, vol. 54, pp. 187-197, 2015.

[11] Z. Zhao, W. Chen, X. Wu, P. C. Y. Chen, and J. Liu, "LSTM network: a deep learning approach for short-term traffic forecast," IET Intelligent Transport Systems, vol. 11, no. 2, pp. 68-75, 2017.

[12] D. Kang, Y. Lv, and Y. Y. Chen, "Short-term traffic flow prediction with LSTM recurrent neural network," in Proceedings of the 2017 IEEE 20th International Conference on Intelligent Transportation Systems (ITSC), pp. 1-6, IEEE, Yokohama, Japan, October 2017.

[13] H. Zou, Y. Wu, H. Zhang, and Y. Zhan, "Short-term traffic flow prediction based on PCC-BiLSTM," in Proceedings of the 2020 International Conference on Computer Engineering and Application (ICCEA), pp. 489-493, IEEE, Guangzhou, China, March 2020.

[14] J. Wang, F. Hu, and L. Li, "Deep bi-directional long shortterm memory model for short-term traffic flow prediction," in Proceedings of the International Conference on Neural Information Processing, pp. 306-316, Springer, Guangzhou, China, November 2017.

[15] T. Sun, C. Yang, K. Han, W. Ma, and F. Zhang, "Bidirectional spatial-temporal network for traffic prediction with multisource data," Transportation Research Record: Journal of the Transportation Research Board, vol. 2674, no. 8, pp. 78-89, 2020.

[16] S. Siami-Namini, N. Tavakoli, and A. S. Namin, "The performance of LSTM and BiLSTM in forecasting time series," in Proceedings of the 2019 IEEE International Conference on Big Data (Big Data), pp. 3285-3292, IEEE, Los Angeles, CA, USA, December 2019.

[17] R. Abduljabbar, H. Dia, S. Liyanage, and S. A. Bagloee, "Applications of artificial intelligence in transport: an overview," Sustainability, vol. 11, no. 1, p. 189, 2019.

[18] Y. Duan, Y. Lv, and F. Y. Wang, "Travel time prediction with LSTM neural network," in Proceedings of the 2016 IEEE 19th International Conference on Intelligent Transportation Systems (ITSC), pp. 1053-1058, IEEE, Rio de Janeiro, Brazil, November 2016.

[19] G. Song, M. Shuai, K. Xie, and X. Ma, "An on-road wireless sensor network approach for urban traffic state monitoring," in Proceedings of the 2008 11th International IEEE Conference on Intelligent Transportation Systems, pp. 1195-1200, IEEE, Beijing, China, October 2008.

[20] R. Lund, "Time series analysis and its applications: with R examples," Journal of the American Statistical Association, vol. 102, no. 479, p. 1079, 2007.

[21] M. G. Karlaftis and E. I. Vlahogianni, "Memory properties and fractional integration in transportation time-series," Transportation Research Part C: Emerging Technologies, vol. 17, no. 4, pp. 444-453, 2009.

[22] G. Fusco, C. Colombaroni, and N. Isaenko, "Short-term speed predictions exploiting big data on large urban road networks," Transportation Research Part C: Emerging Technologies, vol. 73, pp. 183-201, 2016.

[23] C. Chen, J. Hu, Q. Meng, and Y. Zhang, "Short-time traffic flow prediction with ARIMA-GARCH model," in Proceedings of the 2011 IEEE Intelligent Vehicles Symposium (IV), pp. 607-612, Baden-Baden, Germany, June 2011. 
[24] J. Guo, W. Huang, and B. M. Williams, "Adaptive Kalman filter approach for stochastic short-term traffic flow rate prediction and uncertainty quantification," Transportation Research Part C: Emerging Technologies, vol. 43, pp. 50-64, 2014.

[25] M. Lippi, M. Bertini, and P. Frasconi, "Short-term traffic flow forecasting: an experimental comparison of time-series analysis and supervised learning," IEEE Transactions on Intelligent Transportation Systems, vol. 14, no. 2, pp. 871-882, 2013.

[26] J. Morton, T. A. Wheeler, and M. J. Kochenderfer, "Analysis of recurrent neural networks for probabilistic modeling of driver behavior," IEEE Transactions on Intelligent Transportation Systems, vol. 18, no. 5, pp. 1289-1298, 2016.

[27] H. Shao and B. H. Soong, "Traffic flow prediction with long short-term memory networks (LSTMs)," in Proceedings of the 2016 IEEE Region 10 Conference (TENCON), pp. 2986-2989, IEEE, Singapore, November 2016.

[28] Q. Zhaowei, L. Haitao, L. Zhihui, and Z. Tao, "Short-term traffic flow forecasting method with MB-LSTM hybrid network," IEEE Transactions on Intelligent Transportation Systems, pp. 1-11, 2020.

[29] Z. Cui, R. Ke, Z. Pu, and Y. Wang, "Deep bidirectional and unidirectional LSTM recurrent neural network for networkwide traffic speed prediction," 2018, https://arxiv.org/abs/ 1801.02143 .

[30] M. Lu, J. Pang, and J. Li, "DeepBSTN: a deep bidirection network model for urban traffic prediction," in Proceedings of the 2019 5th International Conference on Big Data Computing and Communications (BIGCOM), pp. 1-6, IEEE, QingDao, China, August 2019.

[31] K. Yeon, K. Min, J. Shin, M. Sunwoo, and M. Han, "Egovehicle speed prediction using a long short-term memory based recurrent neural network," International Journal of Automotive Technology, vol. 20, no. 4, pp. 713-722, 2019.

[32] Y. Chen, Y. Chen, and B. Yu, "Speed distribution prediction of freight vehicles on mountainous freeway using deep learning methods," Journal of Advanced Transportation, vol. 2020, Article ID 8953182, 14 pages, 2020.

[33] W. Wang, H. Zhang, T. Li et al., "An interpretable model for short term traffic flow prediction," Mathematics and Computers in Simulation, vol. 171, pp. 264-278, 2020.

[34] M. Farahani, M. Farahani, M. Manthouri, and O. Kaynak, "Short-term traffic flow prediction using variational LSTM networks," 2020, https://arxiv.org/abs/2002.07922.

[35] J. Zhou, H. Chang, X. Cheng, and X. Zhao, "A multiscale and high-precision LSTM-GASVR short-term traffic flow prediction model," Complexity, vol. 2020, Article ID 1434080, 17 pages, 2020.

[36] P. Poonia and V. K. Jain, "Short-term traffic flow prediction: using LSTM," in Proceedings of the 2020 International Conference on Emerging Trends in Communication, Control and Computing (ICONC3), pp. 1-4, IEEE, Sikar, India, February 2020.

[37] C. Kang and Z. Zhang, "Application of LSTM in short-term traffic flow prediction," in Proceedings of the 2020 IEEE 5th International Conference on Intelligent Transportation Engineering (ICITE), pp. 98-101, Beijing, China, September 2020.

[38] S. Lu, Q. Zhang, G. Chen, and D. Seng, "A combined method for short-term traffic flow prediction based on recurrent neural network," Alexandria Engineering Journal, vol. 60, no. 1, pp. 87-84, 2020.
[39] R. Li, Y. Hu, and Q. Liang, "T2F-LSTM method for long-term traffic volume prediction," IEEE Transactions on Fuzzy Systems, vol. 28, no. 12, 2020.

[40] H. Dia, "An object-oriented neural network approach to short-term traffic forecasting," European Journal of Operational Research, vol. 131, no. 2, pp. 253-261, 2001.

[41] H. Dia and G. Rose, "Development and evaluation of neural network freeway incident detection models using field data," Transportation Research Part C: Emerging Technologies, vol. 5, no. 5, pp. 313-331, 1997.

[42] D. T. Larose and C. D. Larose, Discovering Knowledge in Data: an Introduction to Data Mining, Vol. 4, John Wiley \& Sons, Hoboken, NJ, USA, 2014.

[43] K. Yeon, K. Min, J. Shin, M. Sunwoo, and M. Han, "Egovehicle speed prediction using a long short-term memory based recurrent neural network," International Journal of Automotive Technology, vol. 20, no. 4, pp. 713-722, 2019.

[44] C. Sutandi and H. Dia, "Performance evaluation of an advanced traffic control system in a developing country," in Proceedings of the 6th EASTS Conference, vol. 5, pp. 15721584, Bangkok, Thailand, September 2005.

[45] S. Panwai and H. Dia, "Development and evaluation of a reactive agent-based car following model," in Proceedings of the Intelligent Vehicles and Road Infrastructure Conference (IVRI '05), Melbourne, Australia, February 2005.

[46] K. Thomas, H. Dia, and N. Cottman, "Simulation of arterial incident detection using neural networks," in Proceedings of the 8th World Congress on Intelligent Transport Systems, Sydney, Australia, 2001. 